4

5

\title{
Nanchangmycin regulates FYN, FAK and ERK to control the fibrotic activity of hepatic stellate cells
}

Wenyang $\mathrm{Li}^{1,2}$, Jennifer Y. Chen ${ }^{1,2}$, Cheng Sun ${ }^{1,2}$, Robert P. Sparks ${ }^{1,2}$, Lorena Pantano ${ }^{3}$, Raza-Ur Rahman ${ }^{1,2}$, Sean P. Moran ${ }^{1,2}$, Joshua V. Pondick ${ }^{1,2}$, Rory Kirchner ${ }^{3}$, David Wrobel ${ }^{2}$, Michael Bieler ${ }^{5}$, Shannan J. Ho Sui ${ }^{3}$, Julia F. Doerner ${ }^{5}$, Jörg F. Rippmann ${ }^{5}$, Alan C. Mullen ${ }^{1,2,4^{*}}$

1. Gastrointestinal Unit, Massachusetts General Hospital, Boston, MA, USA

2. Harvard Medical School, Boston, MA, USA

3. Harvard T.H. Chan School of Public Health, Boston, MA, USA

4. Harvard Stem Cell Institute, Cambridge, MA, USA

5. Boehringer Ingelheim Pharma GmbH \& Co. KG, Biberach a.d. Riss, Germany

* Corresponding author. Thier 306B, 55 Fruit Street, Massachusetts General Hospital, Boston, MA 02114, USA. Email: acmullen@mgh.harvard.edu

Current Addresses:

Wenyang Li: College of Chemistry and Molecular Engineering, Peking University, Beijing, 100871, China

Jennifer Y. Chen: Department of Medicine, University of California, San Francisco, San Francisco, CA 94115, USA; Liver Center, Department of Medicine, University of California, San Francisco, San Francisco, CA 94143, USA 


\section{Abstract}

31 Chronic liver injury causes fibrosis, characterized by the formation of scar tissue resulting from excessive accumulation of extracellular matrix (ECM) proteins. Hepatic stellate cell (HSC) myofibroblasts are the primary cell type responsible for liver fibrosis, yet there are currently no therapies directed at inhibiting the activity of HSC myofibroblasts. To search for potential antifibrotic drugs, we performed a high-throughput compound screen in primary human HSC myofibroblasts and identified 19 small molecules that induce HSC inactivation, including the polyether ionophore nanchangmycin (NCMC). NCMC induces lipid re-accumulation while reducing collagen expression, deposition of collagen in the extracellular matrix, cell proliferation, and migration. We find that NCMC increases cytosolic $\mathrm{Ca}^{2+}$ and reduces the phosphorylated protein levels of FYN, FAK, ERK1/2, HSP27 and STAT5B. Further, depletion of each of these kinases suppress COL1A1 expression. These studies reveal a signaling network triggered by NCMC to inactivate HSC myofibroblasts and reduce expression of proteins that compose the fibrotic scar. The identification of the antifibrotic effects of NCMC and the pathways by which NCMC inhibits fibrosis provides new tools and therapeutic targets to combat the development and progression of liver fibrosis. 


\section{Introduction}

Chronic liver disease and cirrhosis are the 11th leading cause of mortality in the United States, accounting for over 40,000 deaths annually (Murphy et al., 2021). Liver injuries, including those caused by viral infection, excessive alcohol intake, and nonalcoholic steatohepatitis can lead to fibrosis, the accumulation of abnormal scar tissue, in the liver (Bataller \& Brenner, 2005). If left unchecked, liver fibrosis can progress to cirrhosis and end-stage liver disease (Bataller \& Brenner, 2005). HSC myofibroblasts are the primary cell type responsible for liver fibrosis (Friedman et al., 1985; Mederacke et al., 2013). HSCs reside in the perisinusoidal space and represent $5-8 \%$ of total cells in the liver. In their quiescent, nonfibrotic state, they store vitamin A as retinol ester in lipid droplets (Geerts, 2001). In response to chronic liver injury, HSCs are activated and trans-differentiate into HSC myofibroblasts, characterized by the loss of lipid droplets, increased contractility, and secretion of ECM proteins, leading to fibrosis (Bataller \& Brenner, 2005; Friedman, 2008).

Resolution of liver fibrosis has been observed when the source of liver injury is removed, such as in patients with successful antiviral therapy against hepatitis B or C (Benyon \& Iredale, 2000; Bonis et al., 2001; Falize et al., 2006). Two mechanisms can contribute to the reduction of activated HSC myofibroblasts during resolution of liver fibrosis - apoptosis of activated HSC myofibroblasts and reversion of HSC myofibroblasts to a more quiescent phenotype (Friedman, 2008). With regression of fibrosis, $40-50 \%$ of HSC myofibroblasts revert to an inactive state in vivo, which is associated with reduced collagen expression (Kisseleva et al., 2012; Troeger et al., 2012). These encouraging observations suggest that liver fibrosis is reversible and targeting HSC myofibroblasts to induce an inactive phenotype may serve as a therapeutic approach to treat patients with liver fibrosis.

Despite many efforts to understand HSC plasticity and target HSC myofibroblasts, there are currently no FDA-approved therapies directed at inhibiting the activity of HSC myofibroblasts. 
In our previous studies, we developed a novel small molecule screen to identify compounds that promote HSC inactivation (Chen et al., 2017). In a pilot screen, this approach revealed the antifibrotic effects of tricyclic antidepressants (TCAs). In mechanistic studies, we identified that TCAs inhibit the enzyme acid ceramidase (aCDase). In subsequent studies, we demonstrated that inhibiting aCDase regulates YAP/TAZ-mediated HSC inactivation and reduces fibrogenesis in mouse models and in human precision cut liver slices (Alsamman et al., 2020).

82

Here, we expanded our screen approximately ten-fold to include 15,867 experimental wells and developed a secondary screen to evaluate primary hits. We find that nanchangmycin (NCMC), a polyether ionophore, promotes HSC inactivation. Furthermore, we demonstrate that NCMC decreases proliferation, migration, and assembly of collagen fibers in the extracellular matrix. In additional mechanistic studies, we show that multiple kinases and signaling pathways are involved in mediating the impact of NCMC on HSC activities, including the FYN, FAK and ERK pathways. Taken together, this study defines NCMC as a potent antifibrotic compound that inactivates HSC myofibroblasts and highlights the FYN, FAK and ERK pathways as potential downstream targets to inhibit liver fibrosis.

Results

A high-throughput small molecule screen identifies compounds that inactivate human hepatic stellate cell myofibroblasts

To identify small molecules that induce reversion of HSC myofibroblasts to an inactive phenotype, we screened 24 compound libraries consisting of 15,867 experimental wells using a high-throughput method to quantify lipid droplet accumulation as an indicator of HSC inactivation (Chen et al., 2017) (Figure 1A, Supplementary Table 1, and Supplementary Table 2). Activated HSCs were seeded in 384-well plates, treated with compounds for 48 hours, 
103 fixed, and stained with Bodipy, a fluorescent lipid dye, to analyze the accumulation of lipid

104 droplets as a marker of HSC inactivation. DMSO and nortriptyline were included as negative

105 and positive controls, respectively on each plate. A scaled value was calculated for each

106 experimental well based on the average percentage of Bodipy-positive cells, toxicity, and

107 reproducibility and was normalized according to negative and positive controls on the same

108 plate to minimize plate-specific effects.

110 Experimental wells with a scaled value higher than 0.85 , a cutoff set as the top 10 th percentile

111 of nortriptyline-treated wells on the same plate, were defined as hits. To avoid losing potential

112 hits due to plate-specific effects, the top three experimental wells with the highest scaled

113 values on each plate were also included as hits even if their scaled values did not meet the

1140.85 cutoff. A total of 711 experimental wells, containing 464 different compounds, met these criteria and were identified as primary hits (Figure 1B and Supplementary Table 3).

117 To further narrow the candidate list for secondary screening, the 711 hits were separated into 102 clusters based on their chemical structure, with an average cluster size of seven compounds. The highest scoring hit with the most common structure within each cluster was selected as the representative compound for the cluster. Among the 102 representative compounds, ten compounds were removed because they contained pan assay interference structures (Baell \& Nissink, 2018) or were themselves frequently identified as hits in screens. One compound was removed because it was a TCA, and we have previously demonstrated 124 that TCAs target the sphingolipid pathway to inhibit HSC activity (Chen et al., 2017). One additional compound was removed because it had the same molecular formula as another selected hit (Supplementary Table 4). In addition to representative compounds selected from each cluster, 50 compounds with high scaled values or promising structures were also selected. In total, 140 unique compounds were included in the secondary screen (Figure $1 \mathrm{~A}$ and Supplementary Table 5). 
Development of a secondary screening assay in primary human HSCs

Activated HSCs are characterized by increased expression of $\alpha$-SMA (encoded by ACTA2) and type 1 collagen (encoded by COL1A1) (Bataller \& Brenner, 2005; Friedman, 2008). We developed a high-throughput secondary assay to quantify ACTA2 and COL1A1 mRNA levels as indicators of HSC activity. HSCs were treated with compounds for 48 hours and then lysed for multiplexed qRT-PCR to quantify the house-keeping reference mRNA in the same well as ACTA2 or COL1A1. Since a proper reference mRNA is critical for qRT-PCR based assays, we evaluated 18 housekeeping genes, consisting of seven commonly used genes as well as eleven reference genes identified from the literature (Eisenberg \& Levanon, 2013). We first analyzed RNA sequencing data from HSCs under multiple conditions (Chen et al., 2017). Among these candidates, GUSB, POLR2A, EMC7, VCP, PSMB2 and VPS29 showed the lowest standard deviation ( 0.15 or less). Further comparison of expression of these genes in inactivated HSCs (induced by the addition of nortriptyline or ceramide (Chen et al., 2017)) and culture-activated HSCs revealed that GUSB, POLR2A, EMC7 and PSMB2 had the least fold change in expression (10\% or less upon HSC inactivation). Thus, we selected these four reference mRNAs for further evaluation. GAPDH, which is used routinely as a reference control, was also included for comparison (Figure 1 - figure supplement $1 \mathrm{~A}$ ). We quantified expression using qRT-PCR in HSC cDNA samples reverse-transcribed from equal amounts of total RNA. PSMB2, which encodes proteasome 20 S subunit beta 2, showed the least variation as indicated by standard deviation and was chosen as the reference mRNA for the secondary qRT-PCR-based screen (Figure 1 - figure supplement 1B).

qRT-PCR was performed to quantify ACTA2, COL1A1 and PSMB2 mRNA levels in each sample. Relative fold changes were calculated compared to DMSO control. We defined the following as criteria for compound advancement: 1 . Fold change of COL1A1 was reduced to less than 0.5 of DMSO control (FDR<0.05); 2. Fold change of ACTA2 was reduced to less than 0.5 of DMSO (FDR<0.05); 3. Averaged PSMB2 expression was between $0.2-2.0$ of 
159 DMSO (Figure 1C and Supplementary Table 5). This last criterion was added to avoid

160 selecting compounds where large changes in PSMB2 expression made it difficult to interpret

161 changes in ACTA2 and COL1A1 expression. Of the 140 compounds, a total of 44 compounds

162 met all three criteria. Five compounds were not commercially available, and 39 compounds

163 were advanced for further analysis.

164

165 Next, we evaluated dose response curves (DRCs) for each compound at eight different concentrations, from $10 \mathrm{pM}$ to $10 \mu \mathrm{M}$, using a Bodipy lipid accumulation assay similar to that employed in the primary screen. Dose response curves were scored blindly by three researchers (Supplementary Table 6 and Methods), and nortriptyline served as a reference. Of the 39 compounds, 19 received an average score that was the same as or higher than nortriptyline controls (Figure 1D, Figure 1 - figure supplement 2, and Table 1).

Nine compounds had a DRC score less than 2 and an EC50 less than $5 \mu \mathrm{M}$ and were considered the highest priority. Two subgroups of compounds were identified within this group based on similar bioactivity - histone deacetylase inhibitors (HDACls), including trichostatin A and abexinostat, and Na/K-ATPase inhibitors, including ouabain, digitoxigenin, and digoxin. Histone deacetylases are linked to a variety of fibrotic disorders, including liver fibrosis (Pang \& Zhuang, 2010). HDACls, such as MC1568 and Valproate, have been reported to reduce HSC activation and alleviate liver fibrosis in animal models (Yoon et al., 2019). The presence of HDACls in our final candidate list supports the validity of our screening approach in identifying potential liver fibrosis inhibitors. Na/K-ATPase activity may play a role in nonalcoholic fatty liver disease (Sodhi et al., 2017), but it is not clear how Na/K-ATPases regulate HSC activity and liver fibrosis. Due to the toxicity and narrow therapeutic dose range of cardiac glycosides, which limit their potential application in treatment of liver fibrosis, we decided not to pursue further evaluation of this group of compounds. Nanchangmycin (NCMC), a natural product of Streptomyces nanchangensis, is a polyether insecticidal antibiotic (Sun et al., 2002) and is one of the most potent hits. Studies of NCMC are limited, but it has been shown to have 
a broad spectrum of antiviral activity against diverse arboviruses (Rausch et al., 2017) and potentially SARS-CoV-2 infection (Dittmar et al., 2020; Li et al., 2020; Svenningsen et al., 2020). It also suppresses breast cancer stem cell activity and inhibits growth of breast cancer and multiple myeloma cells (Huang et al., 2018; Xu et al., 2020). The cellular targets of NCMC remain largely unknown, and its effect on HSC activation and liver fibrosis has not been investigated. Therefore, we selected this compound to further validate its function in regulating HSC activity and explore its mechanism of action.

Nanchangmycin induces lipid accumulation in both human and mouse primary HSCs

197 First, we confirmed the effect of NCMC on lipid accumulation in primary HSCs isolated from four human donors of different age, gender and race (donor information provided in Materials and Methods). We observed that NCMC treatment significantly increased lipid droplet accumulation in primary HSC lines compared to controls (Figure 2A), consistent with HSC inactivation. Compared to nortriptyline (Chen et al., 2017), NCMC exhibited higher potency in HSCs from all four donors, where 100-300 nM of NCMC exhibited similar effects on lipid droplet accumulation to $10 \mu \mathrm{M}$ nortriptyline (Figure 2B and Figure 2 - figure supplement 1 ). As we switched to NCMC from a different source with higher purity, we found that the new NCMC stock has a lower EC50 in HSCs from human donors and HSCs from C57BL/6 mice (Figure 2C-D and Figure 2 - figure supplement 2). These results show that NCMC induces lipid accumulation in both human and murine HSCs.

NCMC inhibits fibrotic gene expression in HSCs

211 We next quantified the effect of NCMC on ACTA2 and COL1A1 expression in multiple primary human HSC lines. NCMC treatment reduced both ACTA2 and COL1A1 levels at $100 \mathrm{nM}$ or

213 higher (Figures 3A-B). We also observed a dose-dependent effect of NCMC on Acta2 and 214 Col1a1 expression in murine HSCs at day 2 (Figure 3C). To investigate how NCMC affects 
215 the level of collagen deposited into the ECM, we performed the scar-in-a-jar assay to 216 accelerate the process of ECM deposition with addition of molecular crowding reagents and 217 TGF- $\beta$ (Chen et al., 2009; Good et al., 2019). NCMC treatment significantly decreased 218 collagen staining intensity and fiber area (Figures 3D-E). In addition to two-dimensional (2D) 219 cell culture models, we also tested NCMC's effect on COL1A1 expression in spheroids consisting of primary human HSCs and primary rat hepatocytes. Both the basal expression of COL1A1 and TGF- $\beta$-induced COL1A1 expression were significantly reduced by NCMC treatment (Figure 3F).

HSCs were next treated with NCMC and analyzed by RNA-sequencing analysis, which revealed that NCMC broadly affects genes associated with fibrosis. Among the top gene sets negatively enriched in the NCMC-treated group were ECM-related signatures, including ECM structural constituent and collagen-containing ECM, as well as signatures relevant to migration, including contractile fibers. Of note, genes associated with oligosaccharide lipid intermediate biosynthetic process were positively enriched, possibly contributing to the re-accumulation of lipid droplets (Figure 3G and Supplementary Table 7). We compared the RNA sequencing data with a canonical HSC gene signature (Zhang et al., 2016), an HSC-specific signature that is highly and uniquely expressed in HSCs and correlates with the extent of fibrosis (Zhang et al., 2016), and the liver cirrhosis signature from Disgenet database (Piñero et al., 2020). We observed that these signatures were significantly negatively enriched (Figure 3 - figure supplement 1 and Supplementary Table 8). Visualization of the canonical HSC gene signature (Zhang et al., 2016) also demonstrated that many genes that promote liver fibrosis, including those that encode collagens, TGF- $\beta$, and PDGF pathway components, are inhibited by NCMC triggers a global change in HSC gene expression, resulting in a decrease in profibrotic activity. 
243 In addition to secretion of ECM proteins, activated HSCs demonstrate enhanced migration

244 capabilities (Hernandez-Gea \& Friedman, 2011). Thus, we performed transwell migration and 245 scratch wound healing assays to evaluate how NCMC affects HSC migration. HSCs were pre246 treated with NCMC for two days before seeding in cell culture inserts with permeable 247 membranes. After 6-24 hours, HSCs that migrated through the membrane were stained and counted. NCMC treatment significantly reduced the number of cells that migrated through the membrane compared to DMSO controls (Figure 4A).

We also examined the wound healing capability of HSCs. HSCs were seeded onto plates containing inserts that block cells from accessing and attaching to a strip at the bottom of the well. After removal of the insert, DMSO or $1 \mu \mathrm{M}$ NCMC was added, and HSCs filled the gap or "wound" field through migration and proliferation. After 30 hours of treatment, the DMSOtreated control cells closed the gap, whereas the gap remained for NCMC-treated cells (Figure 4B). While inhibition of HSC migration by NCMC is likely the major contributor to the difference observed between NCMC treatment and controls at shorter time points, such as 6 hours for the transwell assay, the difference at longer time points could be attributed to reductions in both migration and proliferation.

We next determined how NCMC affects HSC proliferation. We treated HSCs isolated from two human donors with DMSO control and increasing concentrations of NCMC and counted Hoechst-stained nuclei on five consecutive days. NCMC treatment reduced cell proliferation at concentrations of $25 \mathrm{nM}$ and higher, and this effect on proliferation was first evident on day (Kisseleva et al., 2012), and we evaluated the contribution of apoptosis to the effect of NCMC on day 2. Based on the analysis of Annexin $\mathrm{V}$ and propidium iodide (PI) by flow cytometry, NCMC treatment only showed a small increase in the percentage of apoptotic cells in one of two donor HSC lines at $1 \mu \mathrm{M}$ and no increase at 100nM. There was also a small increase in 
271 the proliferation marker Ki-67 and PI showed that NCMC treatment increased the percentage

272 of G0 quiescent cells (Figure 4E and Figure 4 - figure supplement 1). Taken together, these

273 results suggest that HSCs enter a more quiescent-like, non-proliferative state upon NCMC

274 treatment, and apoptosis was only observed in a small fraction of cells.

275

Modulation of $\mathrm{Ca}^{2+}$ signaling may contribute to the effect of NCMC on HSC inactivation

278 NCMC is a polyether ionophore and has been shown to increase cytosolic $\mathrm{Ca}^{2+}$ in a cancer 279 cell line (Huang et al., 2018). We examined the activity of NCMC as a calcium ionophore in HSCs. We loaded HSCs with a fluorescent $\mathrm{Ca}^{2+}$ indicator (Fluo-4 NW) and treated cells with NCMC. lonomycin and thapsigargin were included as positive controls (Jones \& Sharpe, 1994; Morgan \& Jacob, 1994). At $10 \mu \mathrm{M}, \mathrm{NCMC}$ increased cytosolic $\mathrm{Ca}^{2+}$ as did ionomycin and thapsigargin (Figure 4F). We also performed the same analysis with ethylene glycol tetraacetic acid (EGTA) to chelate $\mathrm{Ca}^{2+}$ and eliminate any contribution from extracellular $\mathrm{Ca}^{2+}$ during the assay. Similar to the effect observed with thapsigargin (Ribeiro et al., 2018), the increase of cytosolic $\mathrm{Ca}^{2+}$ in response to NCMC was not sensitive to EGTA (Figure 4 - figure supplement $2 \mathrm{~A}$ ), suggesting that the immediate increase of cytosolic $\mathrm{Ca}^{2+}$ following $\mathrm{NCMC}$ treatment is due to release of calcium from intracellular stores. Analysis of a dose response of NCMC demonstrated an increase in cytosolic $\mathrm{Ca}^{2+}$ at concentrations as low as $10 \mathrm{nM}$ (Figure $4 \mathrm{G}$ and Figure 4 - figure supplement 2B).

NCMC reduces COL1A1 expression in HSCs through the FYN pathway

294 Calcium signaling regulates mitogen-activated protein kinases and non-receptor tyrosine kinases (Filvaroff et al., 1990; Rusanescu et al., 1995; Xia et al., 1996), and we analyzed a kinase array to define kinase signaling molecules modulated by NCMC. HSCs were treated with DMSO or $1 \mu \mathrm{M} \mathrm{NCMC}$ for 1 and 18 hours (Figure $5 \mathrm{~A}$ ). Among the 45 proteins tested, 
We selected HSP27 (HSPB1), ERK1/2 (MAPK3/1), STAT5A/B, and FAK (PTK2) to study

further in addition to FYN because 1) they also showed decreased phosphorylation at 18 hours,

and 2) genes encoding these products are expressed at a relatively high level in HSCs, as indicated from RNA sequencing data, suggesting that these may also be potential mechanistic targets of NCMC in HSCs.

304

To further investigate the role of these seven kinases in human HSCs, we depleted each kinase using pooled siRNAs in human HSCs from three donors. We observed a consistent reduction of COL1A1 with depletion of FYN, HSP27, ERK1, ERK2 and STAT5B (Figures 5B-

C, Figure 5 - figure supplement 1), suggesting that each kinase contributes to regulation of COL1A1 expression.

Among the kinases that consistently reduced COL1A1 expression, the reduction of FYN phosphorylation at Y420 was the most prominent following one hour of NCMC treatment, indicating that FYN may mediate the immediate response to NCMC in HSCs. Therefore, we further investigated FYN activity. Western blot of FYN revealed two bands -- the upper band was reduced in HSCs treated with NCMC, while the lower band showed little change (Figure 6 - figure supplement 1 A). Both bands were reduced with depletion of FYN (Figure 6 - figure supplement 2), suggesting that both products are encoded by FYN mRNA. FYN phosphorylation was not directly evaluated because antibodies that uniquely recognize phosphorylated FYN are not available. We also probed with a phospho-Src family antibody, which recognizes phosphorylated FYN and other Src family proteins (Figure 6 - figure supplement 1B). Two bands of approximately the same size are observed with FYN antibody and pSrc antibody, suggesting that both bands may represent phosphorylated FYN, while it is the product in the upper band that is affected by NCMC treatment. QPCR analysis of NCMCtreated HSCs showed that FYN mRNA level was not affected (Figure 6 - figure supplement 3), further indicating that NCMC regulates FYN through a post transcriptional mechanism. 
Both depletion of FYN using two different siRNA duplexes (Figure 6A-B) and treatment with 1-Naphthyl PP1, an inhibitor of v-Src, FYN, and ABL (Figure 6C), significantly reduced COL1A1 mRNA level in HSCs. Collagen deposition in the ECM was also impaired by FYN depletion, as indicated by the reduced collagen intensity and fiber area in the scar-in-a-jar assay (Figures 6D). In addition, ectopic expression of a dominant negative Y213A FYN mutant (Kaspar \& Jaiswal, 2011) reduced COL1A1 expression in HSCs but did not further decrease COL1A1 level in NCMC-treated cells, suggesting that FYN inhibition is likely to be downstream of NCMC (Figure 6E). Lastly, expression of dominant negative FYN in HSCs resulted in a decrease in phosphorylated ERK (Figure 6F), suggesting that FYN may crosstalk with the ERK pathway to exert its function.

ERK1/2 and FAK regulate HSC migration

339

Next, we asked if FYN regulates other HSC phenotypes that are observed with NCMC treatment. HSCs were transfected with pooled FYN siRNAs, and after 3 days, cells were seeded for transwell migration assay. As FAK is known to regulate migration of lung fibroblasts (Zhao et al., 2016) and HSCs (Zhao et al., 2017), we included FAK siRNAs as a positive control. While FAK depletion consistently suppressed migration of HSCs isolated from three different human donors, FYN depletion only reduced migration in one HSC line (Figure 7A-B). We also observed that dominant negative FYN promotes phosphorylation of FAK (Figure 6F), while NCMC reduces phosphorylation of FAK (Figure 6 - figure supplement 4), suggesting that NCMC controls FAK phosphorylation and HSC migration through a pathway that is independent of FYN.

We further tested how other kinases affected by NCMC (ERK1/2 and HSP27) regulate HSC migration. Depletion of ERK1/2 consistently reduced migration across different donors, whereas the influence of HSP27 depletion varied across HSCs from different donors (Figure 
7C). These data indicate that NCMC regulates HSC migration through multiple downstream signaling pathways likely targeting FAK and ERK1/2 as the primary paths to inhibit migration.

\section{Discussion}

Liver fibrosis is the major driver of liver failure in all etiologies of chronic liver disease, and the degree of fibrosis is the strongest predictive factor for liver-related mortality (Anstee et al., 2019). Current therapies for liver fibrosis focus on eliminating the underlying etiology. However, there is a lack of effective treatment for several chronic liver diseases, such as non-alcoholic fatty liver disease, which affects one in four people worldwide (Younossi et al., 2016), and primary sclerosing cholangitis (Karlsen et al., 2017). Therefore, there is an urgent need to develop antifibrotic therapies. Activation of HSCs in the setting of chronic liver injury represents a critical event in fibrosis, as activated HSC myofibroblasts are the primary source of collagen production and excessive extracellular matrix deposition (Friedman et al., 1985; Maher \& McGuire, 1990; Mederacke et al., 2013). With evidence that the scarring process in the liver is reversible (Sun et al., 2020) and that HSC myofibroblasts demonstrate plasticity and can revert to an inactive state (Kisseleva et al., 2012; Troeger et al., 2012), there is increasing enthusiasm for development of approaches to promote HSC inactivation as a therapeutic strategy to treat liver fibrosis. Although some therapies under investigation in clinical trials, including PPAR agonists and TGF- $\beta$ inhibitors, are anticipated to promote HSC inactivation, none have yet been recognized as effective antifibrotic agents (Guo \& Lu, 2020).

Therefore, with the goal to identify new antifibrotic compounds and novel pharmacological targets for the treatment of liver fibrosis, we performed a small molecule compound screen using primary human HSC myofibroblasts. Combining high-content microscopy imaging and high-throughput qPCR screening approaches as well as filtering methods that take into consideration both the potency and diversity of the candidates' chemical structures, we screened 15,867 experimental wells and identified 19 candidates. Further studies to 
investigate the compounds identified by the screen will deepen our understanding of HSC biology and allow identification of additional genes and pathways that could be targeted to reduce liver fibrosis.

We focused on NCMC because it strongly induced HSC inactivation, and the activity of NCMC was poorly understood. NCMC belongs to a group of naturally occurring polyether ionophores, which consist of over 120 known members (Huang et al., 2018). Among them, the compound monensin shares a similar chemical structure to NCMC and is also a positive hit in our primary screen. It was grouped in the same chemical cluster as NCMC, but NCMC was selected as the representative compound for this cluster in the screen because NCMC had a higher scaled value (Supplementary Table 4). This group of compounds demonstrates antibacterial, antifungal, antiparasitic, antimalarial, antiviral, anti-inflammatory activities and cytotoxicity in cancer cells (Kevin li et al., 2009). Although some polyether ionophores have been employed as veterinary antibiotics, none have been used as antibiotics in human, possibly due to concerns about toxicity (Huczyński, 2012). Indeed, we observe that NCMC demonstrated substantial cytotoxicity in cell culture at concentrations higher than $10 \mu \mathrm{M}$ (Figure 2 - figure supplement 1). However, given that the EC50 of NCMC in lipid accumulation assay is in the range of $10-300 \mathrm{nM}$ without inducing apoptosis, NCMC or compounds with similar structure may have potential as antifibrotic therapy within optimized therapeutic doses.

402 Despite the extensive study of some polyether ionophores, limited data are available 403 describing the activity and mechanism of action of NCMC in mammalian cells. A screen for 404 bioactive inhibitors of the Otub1/c-Maf axis in multiple myeloma cells demonstrated that NCMC 405 induces c-Maf polyubiquitination and proteasomal degradation in the presence of Otub1 (Xu et al., 2020). A-130-A, a close analog of NCMC, also inhibited the Wnt/ $\beta$-catenin pathway and induced autophagy, and both A-130-A and NCMC increased cytosolic $\mathrm{Ca}^{2+}$ and reactive oxygen species (ROS) as well as enhanced the permeability of the mitochondrial inner membrane to $\mathrm{H}^{+}$and $\mathrm{K}^{+}$(Huang et al., 2018). To examine a wide variety of signaling pathways 
410 that may be affected downstream of NCMC, we performed a phospho-kinase array analysis

411 to measure the phosphorylation of 37 kinases at a total of 43 different sites and the total protein

412 expression of $\beta$-catenin and HSP60. Seven kinases, including FYN, HSP27, ERK1, ERK2,

413 STAT5A, STAT5B, and FAK, were selected and further tested because their phosphorylated

414 protein was reduced by NCMC treatment, and all were relatively abundant in HSCs. When

415 depleted individually in HSCs from multiple donors, most of these kinases reduced COL1A1

416 level consistently (Figure 5B), suggesting that each may play a role in mediating NCMC's

417 effect on collagen expression. It is unclear why STAT5A siRNAs, which effectively depleted

418 STAT5A but also reduced STAT5B mRNA level, did not significantly affect COL1A1

419 expression, considering that reduction of STAT5B alone to a similar level by STAT5B siRNAs

420 did demonstrate an inhibitory effect (Figure 5B-C and Figure 5 - figure supplement 1 ). It is

421 possible that the expression of STAT5B protein is inhibited more efficiently in HSCs

422 transfected with STAT5B siRNAs than those transfected with STAT5A siRNAs despite the

423 similar mRNA levels. STAT5A/B homo- and hetero-dimers could have different individual

424 DNA-binding specificities (Maurer et al., 2019), and the ratio of homo- to hetero-dimers could also affect transcription of the COL1A1 gene.

427 Our investigations of FYN revealed that depletion or inhibition of FYN activity suppresses collagen expression in primary human HSCs and deposition of collagen in the ECM, but regulation via FYN did not explain all the effects observed with NCMC. The regulation of collagen expression by $\mathrm{FYN}$ is in agreement with a recent study demonstrating that FYN depletion and inhibition in the presence of TGF- $\beta$ reduces collagen I expression in immortalized human and rat HSC lines (Du et al., 2020). This study also observed that FYN depletion and inhibition in the presence of TGF- $\beta$ reduced HSC migration (Du et al., 2020), 434 however, our data did not show a consistent effect on migration with depletion of FYN. As cell migration is controlled by a complex signaling network, the difference in the basal activity of 436 the signaling pathways up- or down-stream of FYN may account for the observed differences.

437 In contrast, FAK and ERK1/2 depletion showed a more robust and consistent inhibitory effect 
among all HSC lines tested, suggesting that these kinases may serve as the critical nodes regulating HSC migration. Depletion or inhibition of FYN or ERK suppresses HSC proliferation

440 (Du et al., 2020; Pagès et al., 1993), and FAK regulates proliferation in many cell types (Zhou 441 et al., 2019). Thus, it is likely that modulation of multiple kinases contributes to the anti-fibrotic 442 effect of NCMC, although the involvement of each kinase may vary depending on the cellular context.

445 We found that NCMC increases cytosolic $\mathrm{Ca}^{2+}$. Although it has not been demonstrated experimentally, it is suspected that NCMC may increase cytosolic $\mathrm{Ca}^{2+}$ levels by disrupting $\mathrm{Na}^{+} / \mathrm{Ca}^{2+}$ exchange (Huang et al., 2018). Of note, the $\mathrm{Na}^{+} / \mathrm{K}^{+}$-ATPase inhibitors identified in our screen can also increase cytosolic $\mathrm{Ca}^{2+}$ levels (Tian \& Xie, 2008), and increased cytosolic $\mathrm{Ca}^{2+}$ has been observed to inhibit ERK1/2 in fibroblasts (Bosch et al., 1998; Chuderland et al., 2020; Cook et al., 1997). As a ubiquitous second messenger with wide-ranging physiological roles, cytosolic $\mathrm{Ca}^{2+}$ levels may be a key factor mediating the downstream anti-fibrotic activity of NCMC, but further investigations are needed to unravel the complete signaling cascade.

In summary, this study has identified NCMC as an antifibrotic compound that increases cytosolic $\mathrm{Ca}^{2+}$ and regulates multiple kinases, including FYN, FAK and ERK1/2 to drive the inactivation of HSC myofibroblasts (Figure 7C). Targeting an individual component of this complex network may suppress certain cellular activities and contribute to HSC inactivation, but it may be necessary to synergistically manipulate multiple targets to achieve antifibrotic effects among the general population. By regulating multiple signaling pathways, NCMC confers a more robust impact than observed with inhibition of only one pathway and thus could represent a more effective strategy to halt fibrosis progression.

\section{Materials and Methods}


467 Human primary hepatic stellate cells from donors 1, 2 and 5 were isolated from human 468 nonparenchymal liver cells (NPCs) purchased from Lonza (cat\# HUCNP) as described 469 previously (Chen et al., 2017). Human primary hepatic stellate cells from donors 3 and 4 were 470 purchased as isolated hepatic stellate cells from Lonza (cat\# HUCLS). Donor information is 471 listed below.

$\begin{array}{cccccc}\text { Donor } & \text { Lonza ID } & \text { Age } & \text { Gender } & \text { Race } & \text { BMI } \\ 1 & 4105 & 45 & \text { M } & \text { Caucasian } & 24.2 \\ 2 & 4270 & 35 & \text { M } & \text { Caucasian } & 42.1 \\ 3 & 180761 & 57 & \text { F } & \text { Caucasian } & 23.6 \\ 4 & 182821 & 24 & \text { F } & \text { African American } & 48.8 \\ 5 & 4258 & 51 & \text { M } & \text { African American } & 24.5\end{array}$

472

473 All hepatic stellate cells were cultured in Dulbecco's Modified Eagle Medium (DMEM) with 10\% 474 fetal calf serum (FCS) and 1\% Penicillin/Streptomycin (P/S). The primary lipid accumulation 475 screen and secondary mRNA screen were conducted with HSCs from donor 1 at passage 8, 476 the dose response curve screen was conducted with HSCs from donor 2 at passage 8 or 9 , 477 and all other experiments were conducted with HSCs from donors as indicated at passage 847810.

480 Nanchangmycin (NCMC) was purchased from two sources. The initial confirmation of dose 481 response curves in multiple HSC lines (Figure 2B and Figure 2 - figure supplement 1) were 482 performed with NCMC purchased from Selleck Chemicals (cat\# S1450). All other experiments 483 were performed with NCMC purchased from Adooq (cat\# A10621) for higher purity. 1484 Naphthyl PP1 was purchased from Tocris (cat\# 3063). Thapsigargin (Sigma-Aldrich, cat\# 
T9033) and ionomycin (Biogems, cat\# 5608212) was purchased from Sigma. Stock solutions were made with DMSO.

For each of the 5-day screening cycle, cells were plated on day 1 at 1000 cells/well in 30 $\mu \mathrm{l} /$ well of complete media in 384-well plates using Multidrop Combi (Thermo). On day 3, 100 $\mathrm{nL} /$ well of compounds from the libraries were transferred by a stainless-steel pin array and Seiko compound transfer robot to the assay plates in duplicates. On day 5 , the cells were fixed with 4\% paraformaldehyde (diluted with DPBS from 16\% stock, Electron Microscopy Sciences, cat\# 15710) and incubated at room temperature for $15 \mathrm{~min}$. The cells were washed one time with DPBS and incubated with Bodipy 493/503 (0.25 $\mu \mathrm{g} / \mathrm{mL}$, Invitrogen, cat\# D3922) and Hoechst $(5 \mu \mathrm{g} / \mathrm{mL}$, Invitrogen, cat\# H1399) for $45 \mathrm{~min}$ at room temperature. The plates were washed three times with DPBS, and then $50 \mu \mathrm{l} /$ well DPBS was added before sealing the plates with adhesive foil cover. The plates were imaged using the ImageXpress Micro Confocal (Molecular Devices) at the Institute of Chemistry and Cell Biology (ICCB)-Longwood screening facility. Images of each well were analyzed using MetaXpress software to calculate the percentage of positive cells (the total number of Bodipy positive cells (cutoff was adjusted for each plate) divided by the total cell count).

504

We developed a scoring system to rank the strength of a compound in inducing HSC reversion

506 to the inactive phenotype. A score was calculated as follows: 1. Averaged percent positive 507 cells from duplicates was used to calculate the distance from the baseline of the plate (percentile 75\%), 2. Toxicity was penalized (the distance from the average number of cells in the compound wells to the number of cells in the nortriptyline wells), and 3. Poor reproducibility was penalized (the error of the two points to the average value of the duplicates). The score was then normalized using nortriptyline and DMSO scores for each plate. A new parameter 
512 was calculated termed "Scaled" with the formula: Scaled $=-1^{*}$ (median (nortriptyline)-

513 score)/abs(median (nortriptyline)-median(DMSO)) .

514

515 Consolidation of screening library

516

517 Chemical structures of the screening library were consolidated using the data science

518 workflow software BIOVIA Pipeline Pilot. Protonation states of the structures were

519 standardized, and counter ions were eliminated. We used canonical Simplified Molecular Input

520 Line Entry System (SMILES) as a unique linear textual representation of the chemical

521 structure. This way, the initial 15867 structures could be mapped onto 7696 unique canonical

522 SMILES of which 4329 are represented by a single well in the library and 3367 occur in up to

52319 wells. Multiple occurrences of individual canonical SMILES could be traced to multiple vendors and/or multiple molar concentrations of the individual probes. Using this analysis, the 711 experimental wells defined as hits were determined to represent 464 individual compounds.

Hits were clustered into 102 groups of structurally similar compounds based on Tanimoto similarities using the computational analysis software BIOVIA Pipeline Pilot. The distance to

532 the center of the cluster was calculated for each compound in the cluster using BIOVIA 533 Pipeline Pilot, and the most common structure for each cluster was defined based on this 534 value. The strongest hit with the most common structure for each cluster was selected as the representative for the cluster. Promiscuous bioactive compounds that contain pan assay interference structures (PAINS) (Baell \& Nissink, 2018), or that we identified as frequent hits

537 in screens at ICCB-L were not included for further analysis, as the exhibited bioactivity may 538 be attributed to interference with specific assay readouts and/or nonspecific, intractable 539 mechanism of action (Matlock et al., 2018). Frequent hits were defined as having a positive 
540 hit rate of more than $20 \%$ in screens performed at ICCB-L or more than 10 total positive hits

541 in the database of ICCB-L screens. One additional compound was removed because the

542 molecular formula was the same as another selected compound, and one compound was

543 removed due to similarity in structure to nortriptyline (Supplementary Table 4).

544

Secondary mRNA screen for cherrypicked small molecules

546

547 For each of the 5-day screening cycle, cells were plated in 384-well plates as in primary screen

548 on day 1. Compounds were added on day 3 using a digital non-contact dispenser D300e

549 (Hewlett Packard) in quadruplicate. On day 5, cell lysates for qPCR were prepared using the 550 Cells-to- $\mathrm{C}_{\mathrm{T}}$ 1-Step Taqman Kit (Invitrogen, cat\# A25603) according to manufacturer's instructions. Briefly, cells were incubated with $25 \mu \mathrm{L} /$ well lysis buffer (plus DNase) for 5 minutes at room temperature, and the reaction was stopped by adding $2.5 \mu \mathrm{L}$ stop solution and incubating for 2 minutes at room temperature. $2 \mu \mathrm{L}$ cell lysates were used in the multiplexed qRT-PCRs to measure ACTA2, COL1A1, and PSMB2 mRNA levels. To reduce technical variations, the TaqMan probe for endogenous control gene PSMB2 was VIC-labeled and primer-limited, so that the PSBM2 probe can be multiplexed with FAM-labeled probe for ACTA2 or COL1A1 in the same qRT-PCR. Details for probes are included in the "qPCR analysis" section. The results were analyzed by fitting the data to the following linear models: Ct_ACTA2 Ct_PSMB2 + plate + chemical or Ct_COL1A1 Ct_PSMB2 + plate + chemical. Relative fold changes were calculated from the estimate of each chemical as compared to DMSO control.

Dose response curve screen

The dose response curve screen was performed using an adapted lipid accumulation assay with live human primary HSCs. Briefly, cells were plated at a density of 2500 cells/well in 384well plates. After 24 hours, compounds were added in duplicate at concentrations from 0.001 
to $10 \mu \mathrm{M}$. Nortriptyline $(10 \mu \mathrm{M})$ and DMSO $(0.1 \%)$ served as controls. Cells were incubated with compounds for 24 hours, followed by treatment with Bodipy $(1 \mu \mathrm{g} / \mathrm{ml}$; ThermoFisher, cat\# D3922) and NucLight Rapid Red (final dilution 1:4000; Essen BioSciences, cat\# 4725) for an additional 12 hours to stain lipid droplets and nuclei. Fluorescent signals were measured using an Incucyte S3 system.

573

574 Bodipy stained area and nuclei count were determined selecting two fields per well. The 575 Bodipy-stained area per nuclei count was calculated per field and the mean was determined.

576 The percentage of lipid accumulation (referred to as \% CTL) in response to compound treatment was analyzed as follows: $100 \times$ ((total green area/count [test compound] - total green area/count [mean DMSO]) / (total green area/count [mean Nor] - total green area/count [mean DMSO])). The total green area is a measure of lipid droplet accumulation in $\mu^{2} /$ well, and count indicates the cell (nuclei) number per well.

582 The dose-response measurements were reviewed and scored independently by three 583 researchers based on the following criteria: Priority 1: the percentage of lipid accumulation (\% $584 \mathrm{CTL}$ ) is increased at $1 \mu \mathrm{M}$, the shape suggests a sigmoidal distribution, and at least two concentrations show increased Bodipy staining before the compound becomes toxic; Priority 2: compound treatment at $3.03 \mu \mathrm{M}$ and $10 \mu \mathrm{M}$ (highest concentrations) shows increased Bodipy staining, and \% CTL is at least $70 \%$ at $10 \mu \mathrm{M}$; Priority 3: compound treatment at 3.03 $\mu \mathrm{M}$ and $10 \mu \mathrm{M}$ (highest concentrations) shows increased Bodipy staining, and \% CTL is less than $70 \%$ at $10 \mu \mathrm{M}$; Priority 4: only treatment at the highest concentration $(10 \mu \mathrm{M})$ shows increased Bodipy staining ; Priority 5: the curve is almost flat (no response).

Estimation of EC50 for NCMC 
596

597

598

599

600

601

602

603

604

605

606

607

608

609

610

611

612

613

614

615

616

617

618

619

620

specified, and analyzed by lipid accumulation assay similar to the primary screen. Once the percentage of Bodipy-positive cells was determined, the data were fitted into a sigmoidal four parameter logistic model in Graphpad Prism software to estimate the EC50 of NCMC under each condition.

\section{Fluorescent microscopy}

HSCs were seeded in black-wall 96-well plates (Corning, cat\# 3603) and treated with NCMC at different concentrations as indicated. After 48 hours, plates were fixed with $4 \%$ paraformaldehyde and stained with Bodipy and Hoechst as in the primary lipid accumulation screen. After the last wash, $200 \mu \mathrm{l} /$ well DPBS was added, and plates were imaged using a Nikon A1R confocal microscope.

qPCR analysis

qPCR analysis related to depletion of kinase candidates was performed using lysates prepared with the Cells-to- $\mathrm{C}_{\mathrm{T}}$ 1-Step Taqman Kit similar to the secondary mRNA screen, except that HSCs were seeded in 96-well plates and $50 \mu$ lysis buffer (with DNase) and $5 \mu \mathrm{l}$ stop solution were used. For the other qPCR analyses, RNA samples were prepared using TRIzol (Invitrogen, cat\# 15596026), and the concentrations were quantified using Qubit 3 fluorometer (Invitrogen) and the Qubit RNA BR Assay Kit (Invitrogen, cat\# Q10211) according to manufacturer's instructions. Reverse transcription was performed using iScript gDNA Clear cDNA Synthesis Kit (BIO-RAD, cat\# 1725035) with $1 \mu \mathrm{g}$ total RNA input, and quantitative realtime PCR was performed using TaqMan Universal PCR Master Mix (Applied Biosystems, cat\# 4305719) and TaqMan Real-time PCR Assays for specific genes listed below.

Assay ID 


\begin{tabular}{|c|c|}
\hline Human ACTA2 & Hs00426835_g1 \\
\hline Human COL1A1 & Hs00164004_m1 \\
\hline Human FYN & Hs00176628_m1 \\
\hline Human HSPB1/HSP27 & Hs00356629_g1 \\
\hline Human MAPK1/ERK2 & Hs01046830_m1 \\
\hline Human MAPK3/ERK1 & Hs00385075_m1 \\
\hline Human PSMB2 & Hs01002946_m1 \\
\hline Human PTK2/FAK & Hs01056457_m1 \\
\hline Human STAT5A & Hs00559643_m1 \\
\hline Human STAT5B & Hs00560026_m1 \\
\hline Mouse Acta2 & Mm00725412_s1 \\
\hline Mouse Col1a1 & Mm00801666_g1 \\
\hline Mouse Psmb2 & Mm00449477_m \\
\hline
\end{tabular}

624 To test the effect of NCMC on collagen deposition in ECM, HSCs were seeded in black-wall 625 96-well plates (Corning, cat\# 3603) and treated with DMSO or $100 \mathrm{nM} \mathrm{NCMC}$ for 48 hours in 626 Ficoll medium, i.e. complete medium supplemented with $50 \mu \mathrm{g} / \mathrm{ml}$ L-ascorbic acid 2627 phosphate sesquimagnesium salt hydrate (Sigma, cat\# A8960), $37.5 \mathrm{mg} / \mathrm{ml}$ Ficoll-PM70 628 (Sigma, cat\# F2878), 25 mg/ml Ficoll-PM400 (Sigma, cat\# F4375) and 5 ng/mL TGF- $\beta$ (R\&D 629 Systems, cat\# 240-B-002). To test the effect of FYN depletion on collagen deposition, HSCs 
630 were reverse transfected in 96-well plates with $50 \mathrm{nM}$ non-targeting control siRNA or siRNAs

631 against FYN (see the "RNAi-mediated depletion of genes" section for specific information on

632 siRNAs and transfection reagents). After 48 hours, cells were incubated with Ficoll medium

633 for an additional 48 hours.

634

635 Cells were then fixed with ice-cold methanol for 2 min on ice, washed one time with DPBS and 636 then incubated with primary antibody against collagen type I in DPBS (1:1000, Sigma, cat\# $637 \mathrm{C} 2456)$ at $4{ }^{\circ} \mathrm{C}$ overnight. After three washes with PBS-Tween $(0.05 \% \mathrm{v} / \mathrm{v})$, cells were 638 incubated with donkey anti-mouse Alexa Fluor 488 secondary antibody (1:500, Invitrogen, 639 cat\# A-21202) and Hoechst (1:4000) in DPBS at room temperature for 1 hour. Plates were 640 washed three times with PBS-Tween, and after the final wash, $200 \mu \mathrm{L} /$ well of DPBS was 641 added. Plates were imaged using the ImageXpress Micro Confocal microscope (Molecular 642 Devices) with 10x Plan Apo lens, and collagen fibers were analyzed using a custom module 643 built within the MetaXpress software.

644

Liver spheroid experiment

646

647 Liver spheroids were prepared as previously described (Leite et al., 2016) except spheroids were formed from primary rat hepatocytes (Lonza, cat\# RSCP01) and primary human HSCs.

649 Cells were seeded in ultra-low attachment round bottom 96-well plates (Greiner Bio-One, cat\# 650 650970) at a ratio of 1:2. The cells were incubated in HCM hepatocyte culture media (Lonza, cat\# CC-3198) for one day with orbital shaking to allow the generation of liver spheroids. The spheroids were then treated with DMSO or NCMC for 72 hours with or without TGF- $\beta$ (5 $\mathrm{ng} / \mathrm{mL}$ ). Spheroids were collected, and RNA was extracted to quantify expression of human 
HSCs were treated with DMSO or $1 \mu \mathrm{M}$ NCMC for 48 hours. RNA was extracted using RNeasy Mini kit (Qiagen, cat\# 74104), followed by quality assessment via Agilent 2200 Tape Station. prepared for NCMC treatment, and all samples had an RNA integrity number (RIN) greater cat\# 20020594) and sequenced on a HiSeq2000.

664

For data analysis, reads were quality assessed using the FASTQC $(v 0.11 .8)$ and aligned to the human reference genome (GRCh38_release_37) from GENCODE with Star aligner (v2.7.3) using RSEM (v1.3.1) with default parameters. First, the human reference genome quantify the gene abundance. The output of rsem-calculate-expression gives separately the read count and transcripts per million (TPM) value for each gene.

672

Differential expression analysis

674

675 Differential expression analysis was performed using gene read counts with DESeq2 package ( $v$ 1.32.0) to produce LFC values and corresponding p-values (FDR) applying a BenjaminiHochberg correction for multiple testing. The heatmap was created using normalized gene count values from Deseq2, using $\mathrm{R}$ gplots package heatmap.2 function with row scaling.

Gene set enrichment analysis (GSEA)

Gene set enrichment analysis was performed using the GSEA software downloaded from http://www.gsea-msigdb.org/gsea/index.jsp (Mootha et al., 2003; Subramanian et al., 2005).

684 An expression dataset containing gene name and log2 (fold change) was generated based on 685 the RNA sequencing results and loaded to the software as the input file. The c5.all.v7.4 gene 
matrix was used as the database of gene sets, and gene sets smaller than 10 or larger than

6871000 in size were excluded for the analysis. The canonical HSC gene signature and specific

688 HSC gene signature were obtained from previous publication (Zhang et al., 2016), and the

689 liver cirrhosis signature was downloaded from Disgenet database (Piñero et al., 2020). Among

690 the 44 genes in the canonical HSC signature, 35 were found in our differential expression list.

691 Among the 122 genes in the specific HSC signature, 97 were found in our differential

692 expression list. Among the 103 genes in the liver cirrhosis signature, 69 were found in our

693 differential expression list. These genes were listed in Supplementary Table 8.

694

695

Transwell migration assay

696

697

HSCs were treated with DMSO or $1 \mu \mathrm{M}$ NCMC for 48 hours or transfected with siRNAs for 72

698

hours in 6-well plates. HSCs were then trypsinized and counted to seed at 5,000-10,000 cells

699 per insert depending on donor and assay duration (Corning, cat\# CLS3422) in serum-free

DMEM. Complete medium (with 10\% FBS) was added to the bottom well to induce cell

migration through the pores (diameter: $8 \mu \mathrm{m}$ ) of the membrane at the bottom of the insert.

After the indicated assay time, cells were fixed with $4 \%$ paraformaldehyde at room C0775) for 1 hour. Inserts were washed with DPBS, and the cells that had not migrated through the pores and remained on the upper side of the membrane were removed with cotton swabs. Images were taken using EVOS XL Core microscope with 10x lens under brightfield.

HSCs were plated in CytoSelect 24-well wound healing assay plates with inserts (Cell Biolabs, cat\# CBA-120) at 400,000 cells/well in complete medium. Eighteen hours after plating, inserts were removed to generate a $0.9 \mathrm{~mm}$ wound field, and cells were incubated with complete 
713 medium containing DMSO or NCMC for an additional 30 hours. Images were taken using

714 EVOS FL microscope.

715

716

Proliferation assay

717

718

HSCs were seeded in black-wall 96-well plates (Corning, cat\# 3603) at 3,000 cells/well, and

71918 hours later, DMSO and NCMC at different concentrations as indicated were added with six replicates. One plate was fixed on each day with $4 \%$ paraformaldehyde for five days consecutively and stored at $4^{\circ} \mathrm{C}$ until all plates were ready for staining with Hoechst. ImageXpress Micro Confocal microscope (Molecular Devices) was used for taking four images/well with 10x Plan Apo lens, and MetaXpress software was used for counting the number of nuclei.

725

Apoptosis analysis by flow cytometry

HSCs were treated with DMSO or NCMC at indicated concentrations for 48 hours, followed using FACSAria II (BD Biosciences).

Quiescence/cell cycle analysis by flow cytometry

HSCs were treated with DMSO or NCMC at indicated concentrations for 48 hours, trypsinized, harvested, washed with DPBS, and resuspended in $0.5 \mathrm{~mL}$ DPBS. Cells were fixed by adding $4.5 \mathrm{~mL}$ ice-cold $70 \%$ ethanol in a drop wise manner while vortexing and were then kept at $73820^{\circ} \mathrm{C}$ for at least 2 hours. Cells were washed twice with FACS buffer (DPBS supplemented with $2 \%$ heated-inactivated filtered fetal bovine serum and $1 \mathrm{mM}$ EDTA) before resuspending in FACS buffer at $1 \times 10^{6}$ cells $/ 100 \mu \mathrm{L}$. Cells were then incubated with Ki-67 antibody 
$741(0.25 \mu \mathrm{g} / 100 \mu \mathrm{L}$, clone SolA15, Invitrogen, cat\# 11-5698-82) in the dark for 30 min at room

742 temperature. After incubation, cells were washed twice with FACS buffer, followed by

743 incubation with propidium iodide staining solution (DPBS supplemented with $50 \mu \mathrm{g} / \mathrm{ml}$

744 propidium iodide (Invitrogen, cat\# P3566), 10 $\mathrm{g} / \mathrm{ml}$ RNase (Thermo Scientific, cat\# EN0531)

745 and $2 \mathrm{mM} \mathrm{MgCl}_{2}$ ) for another $20 \mathrm{~min}$ at room temperature before analysis by FACSAria II (BD

746 Biosciences).

747

748 Calcium measurements

749

750 Fluo-4 NW calcium assay starter kit (Invitrogen, F36206) was used to measure cytosolic 751 calcium according to the manufacturer's protocol in the presence and absence of 1 mM EGTA. HSCs were plated on a Corning 96 well UV transparent plate 24 hours prior to analysis. Media was removed and cells were washed with $1 \mathrm{X}$ calcium and magnesium chloride free PBS before adding the dye mix with probenecid with and without EGTA to each well. Plates were covered in aluminum foil and incubated at $37^{\circ} \mathrm{C}$ for 30 minutes. Plates were equilibrated to room temperature for 30 mins prior to analysis. Measurements were performed on a Tecan Infinite M Plex M-200 using I-control 2.0 software from Austria $\mathrm{GmbH}$ to measure fluorescence intensity with excitation at $494 \mathrm{~nm}$ and emission at $516 \mathrm{~nm}$. Readings were performed by loading the plate immediately following treatment with DMSO or compounds at the indicated concentration. All measurements were normalized to time 0 by subtracting the initial value for each well. Based on this approach, the increase in $\mathrm{Ca}^{2+}$ levels measured for ionomycin may be reduced due to a more rapid response to the compound. Measurements were plotted as change in relative fluorescence unit (RFU) at $50 \%$ gain on the $\mathrm{Y}$-axis relative to time 0 in seconds on the X-axis using Graphpad Prism 8.4.3.

765 
HSCs from donor 3 were treated with DMSO or $1 \mu \mathrm{M} \mathrm{NCMC} \mathrm{for} 1$ hour or 18 hours. Cell lysates were prepared and analyzed using the Proteome Profiler Human Phospho-Kinase Array Kit (R\&D Systems, cat\# ARY003B) according to manufacturer's instructions. Scanned

771 films were quantified using ImageJ.

772

RNAi-mediated depletion of gene expression

774

HSCs were reverse transfected with siRNAs as indicated using Dharmafect-1 transfection reagent (Horizon Discovery, cat\# T-2001) according to manufacturer's instructions. For 12well plates, $60 \mu \mathrm{L}$ of $1 \mu \mathrm{M}$ siRNAs were added to $180 \mu \mathrm{L}$ Opti-MEM (Gibco, cat\# 31985070) for the final concentration of $50 \mathrm{nM}$ and then mixed with diluted Dharmafect- 1 in Opti-MEM $(1.2 \mu \mathrm{L}$ Dharmafect-1 in 238.8 $4 \mathrm{~L}$ Opti-MEM). After $30 \mathrm{~min}$, HSCs resuspended in transfection medium (DMEM supplemented with $16 \%$ FCS) were seeded in the wells containing the siRNA/Dharmafect-1 mixture at 70,700 cells $/ \mathrm{mL}$ in $720 \mu \mathrm{L} /$ well medium. Transfection in other plate formats were scaled up or down accordingly based on surface area. Cells were incubated with siRNAs and transfection reagents for 72 hours before analysis, unless indicated otherwise.

785

The siRNAs used in this study are siGENOME SMARTpool siRNAs (Horizon Discovery) for FYN (MQ-003140-04), HSP27 (M-005269-01), ERK1 (M-003592-03), ERK2 (M-003555-04), FAK (M-003164-02), STAT5A (M-005169-02) and STAT5B (M-010539-02) and individual siGENOME siRNAs for FYN (siRNA\#1: D-003140-10, siRNA\#2: D-003140-24).

Cloning, lentivirus packaging and infection

792

793 The cDNA encoding the dominant negative Y213A FYN mutant was amplified from the 794 plasmid pRK5 DN-Fyn (gift from Filippo Giancotti, Addgene plasmid \# 16033) using the 
ATGGGCTGTGTGCAATGTAAGG-3'; reverse primer: 5'- AGC GAATTC

797 TTACAGGTTTTCACCAGGTTGGTAC-3'. The amplified PCR product was digested with Nhel

798 and EcoRI enzymes and inserted into linearized pLJM1 plasmid (gift from David Sabatini, 799 Addgene, plasmid\# 19319). Whole plasmid sequencing was performed to confirm that the DN800 FYN sequence was correct.

801

802 HEK 293 cells were transfected with pLJM1-eGFP or pLJM1-DN-FYN plasmid together with 803 lentivirus packing and envelope plasmids pMD2.G (gift from Didier Trono, Addgene plasmid\# 804 12259) and psPAX2 (gift from Didier Trono, Addgene plasmid\# 12260) using X-tremeGENE 8059 DNA transfection reagent (Roche, cat\# 6365779001) according to manufacturer's 806 instructions. For a $10 \mathrm{~cm}$ dish of 293 cells, $30 \mu \mathrm{L}$ X-tremeGENE 9 reagent, $750 \mathrm{ng}$ psPAX2,

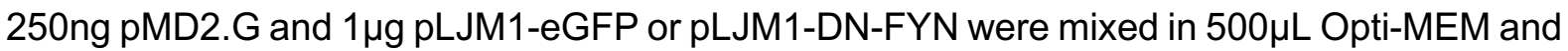
incubated for 15 min before added to culture medium in a drop wise manner. 24 hours later, culture medium was changed, and cells were incubated with fresh regular medium for another 24 hours. Conditioned medium containing virus was then collected and filtered through $0.45 \mu \mathrm{m}$ filters. HSCs were seeded to reach $30-40 \%$ confluency after 18 hours and then infected with viruses. Polybrene (Sigma-Aldrich, cat\# TR-1003-G) was used at a final concentration of $10 \mu \mathrm{g} / \mathrm{mL}$ to enhance infection efficiency.

\section{Western blot}

817 Cells were pelleted and lysed with RIPA buffer (150 mM sodium chloride, $1.0 \%$ Triton X-100, $8180.5 \%$ sodium deoxycholate, $0.1 \%$ SDS (sodium dodecyl sulfate) and $50 \mathrm{mM}$ Tris, $\mathrm{pH} 8.0$ ) 819 supplemented with protease inhibitors (Thermo Scientific, cat\# 87786) and phosphatase 820 inhibitors (Thermo Scientific, cat\# 78420). Cell lysates were centrifuged to remove debris.

821 Protein concentrations were measured using Pierce ${ }^{\mathrm{TM}}$ BCA Protein Assay Kit (Thermo 822 Scientific, cat\# 23227). Bolt ${ }^{\text {TM }}$ LDS Sample Buffer (Invitrogen, cat\# B0007) and Bolt ${ }^{\text {TM }}$ Sample 823 Reducing Agent (Invitrogen, cat\# B0009) were added to cell lysates and the sample mixture 
824 was boiled for 10 min before loading. Bolt ${ }^{\mathrm{TM}} 4$ to $12 \%$ Bis-Tris gels (Invitrogen, cat\#

825 NW04120BOX) were used for electrophoresis, followed by transferring with iBlot 2 Dry Blotting

826 System (Invitrogen, cat\# IB21002S). Membranes were blocked with 1\% BSA (Thermo

827 Scientific, cat\# 37520, for phospho-Src family) or 3\% milk (Lab Scientific, cat\# M0841, for

828 other proteins) at room temperature for 1 hour and incubated with primary antibody at $4^{\circ} \mathrm{C}$ for

829 two days (for FYN) or overnight (for other proteins). Membranes were washed three times with

830 Tris Buffered Saline-Tween (TBST) buffer (Boston BioProducts, cat\# IBB-181-6), incubated

831 with secondary antibody for another 1 hour, washed three times with TBST buffer, and then

832 incubated with SuperSignal ${ }^{\mathrm{TM}}$ West Pico PLUS chemiluminescent substrates (Thermo

833 Scientific, cat\# 34580) for 5 min before exposure to film (Ece Scientific Co, cat\# E3018). The

834 following antibodies were used: FYN antibody (1:1000, Cell Signaling Technology, cat\# 4023),

835 phospho-Src family antibody (1:1000, Cell Signaling Technology, cat\# 6943), HRP-ß-Actin

836 antibody (Santa Cruz Biotechnology, cat\# sc-47778), phospho-p44/42 MAPK (Erk1/2)

837 (Thr202/Tyr204) antibody (1:1000, Cell Signaling Technology, cat\# 4370), and goat anti-rabbit

838 lgG secondary antibody (Invitrogen, cat\# 32460).

839

840

Data availability

841

842 RNA sequencing data produced in this study have been submitted to GEO (GSE180980). The

843 full dataset will be accessible when released.

844

845 Acknowledgements

846 We thank the staff at the ICCB-Longwood Screening Facility, and especially Jennifer Smith

847 and Richard Siu for their assistance in establishing and performing the small molecule screen.

848 We thank members of the Mullen lab in addition to Raymond Chung for helpful discussions,

849 along with colleagues at Boehringer Ingelheim, including Birgit Goetz, Katja Thode, Elfriede

850 Mueller, Manuela Schuler, Nicola Zimmerman for experimental support, and Carine Boustany

851 and Daniela Moutinho Dos Santos for helpful discussions. We also thank the MGH Next Gen 
Sequencing Core, MGH Center for Regenerative Medicine Flow Cytometry Facility, and MGH

Program in Membrane Biology Microscopy Core for their support. This work was funded

through a grant from Boehringer Ingelheim.

855

\section{Competing interests}

857

$\mathrm{MB}, \mathrm{JFR}$, and JFD are employees of Boehringer Ingelheim. ACM receives funding from

Bristol-Myers Squibb and GlaxoSmithKline for unrelated projects and is a consultant for Circ

Bio.

860

\section{References}

862

Alsamman, S., Christenson, S. A., Yu, A., Ayad, N. M. E., Mooring, M. S., Segal, J. M., . . . Chen, J. Y. (2020). Targeting acid ceramidase inhibits YAP/TAZ signaling to reduce fibrosis in mice. Sci Transl Med, 12(557). https://doi.org/10.1126/scitranslmed.aay8798

Anstee, Q. M., Reeves, H. L., Kotsiliti, E., Govaere, O., \& Heikenwalder, M. (2019). From NASH to HCC: current concepts and future challenges. Nat Rev Gastroenterol Hepatol, 16(7), 411-428. https://doi.org/10.1038/s41575-019-0145-7

Baell, J. B., \& Nissink, J. W. M. (2018). Seven Year Itch: Pan-Assay Interference Compounds (PAINS) in 2017-Utility and Limitations. ACS Chem Biol, 13(1), 36-44. https://doi.org/10.1021/acschembio.7b00903

Bataller, R., \& Brenner, D. A. (2005). Liver fibrosis. J Clin Invest, 115(2), 209-218. https://doi.org/10.1172/JCl24282

Benyon, R. C., \& Iredale, J. P. (2000). Is liver fibrosis reversible? Gut, 46(4), 443-446. https://doi.org/10.1136/gut.46.4.443

Bonis, P. A., Friedman, S. L., \& Kaplan, M. M. (2001). Is liver fibrosis reversible? N Engl J Med, 344(6), 452-454. https://doi.org/10.1056/NEJM200102083440610

Bosch, M., Gil, J., Bachs, O., \& Agell, N. (1998). Calmodulin inhibitor W13 induces sustained activation of ERK2 and expression of p21(cip1). J Biol Chem, 273(34), 22145-22150. https://doi.org/10.1074/jbc.273.34.22145

Chen, C. Z., Peng, Y. X., Wang, Z. B., Fish, P. V., Kaar, J. L., Koepsel, R. R., . . Raghunath, M. (2009). The Scar-in-a-Jar: studying potential antifibrotic compounds from the epigenetic to extracellular level in a single well. Br J Pharmacol, 158(5), 1196-1209. https://doi.org/10.1111/j.1476-5381.2009.00387.x

Chen, J. Y., Newcomb, B., Zhou, C., Pondick, J. V., Ghoshal, S., York, S. R., . . Mullen, A. C. (2017). Tricyclic Antidepressants Promote Ceramide Accumulation to Regulate Collagen Production in Human Hepatic Stellate Cells. Sci Rep, 7, 44867. https://doi.org/10.1038/srep44867

Chuderland, D., Marmor, G., Shainskaya, A., \& Seger, R. (2020). Calcium-Mediated Interactions Regulate the Subcellular Localization of Extracellular Signal-Regulated Kinases (ERKs). Cell Physiol Biochem, 54(3), 474-492. https://doi.org/10.33594/000000231

Cook, S. J., Beltman, J., Cadwallader, K. A., McMahon, M., \& McCormick, F. (1997). Regulation of mitogen-activated protein kinase phosphatase-1 expression by 
extracellular signal-related kinase-dependent and Ca2+-dependent signal pathways in

Rat-1 cells. J Biol Chem, 272(20), 13309-13319.

Dittmar, M., Lee, J. S., Whig, K., Segrist, E., Li, M., Jurado, K., . . Cherry, S. (2020). Drug repurposing screens reveal FDA approved drugs active against SARS-Cov-2. bioRxiv, 2020.2006.2019.161042. https://doi.org/10.1101/2020.06.19.161042

Du, G., Wang, J., Zhang, T., Ding, Q., Jia, X., Zhao, X., . . Lu, Y. (2020). Targeting Src family kinase member Fyn by Saracatinib attenuated liver fibrosis in vitro and in vivo. Cell Death Dis, 11(2), 118. https://doi.org/10.1038/s41419-020-2229-2

Eisenberg, E., \& Levanon, E. Y. (2013). Human housekeeping genes, revisited. Trends Genet, 29(10), 569-574. https://doi.org/10.1016/j.tig.2013.05.010

Falize, L., Guillygomarc'h, A., Perrin, M., Lainé, F., Guyader, D., Brissot, P., . . Deugnier, Y. (2006). Reversibility of hepatic fibrosis in treated genetic hemochromatosis: a study of 36 cases. Hepatology, 44(2), 472-477. https://doi.org/10.1002/hep.21260

Filvaroff, E., Stern, D. F., \& Dotto, G. P. (1990). Tyrosine phosphorylation is an early and specific event involved in primary keratinocyte differentiation. Mol Cell Biol, 10(3), 1164-1173. https://doi.org/10.1128/mcb.10.3.1164-1173.1990

Friedman, S. L. (2008). Hepatic stellate cells: protean, multifunctional, and enigmatic cells of the liver. Physiol Rev, 88(1), 125-172. https://doi.org/10.1152/physrev.00013.2007

Friedman, S. L., Roll, F. J., Boyles, J., \& Bissell, D. M. (1985). Hepatic lipocytes: the principal collagen-producing cells of normal rat liver. Proc Natl Acad Sci U S A, 82(24), 86818685. https://doi.org/10.1073/pnas.82.24.8681

Geerts, A. (2001). History, heterogeneity, developmental biology, and functions of quiescent hepatic stellate cells. Semin Liver Dis, 21(3), 311-335. https://doi.org/10.1055/s-200117550

Good, R. B., Eley, J. D., Gower, E., Butt, G., Blanchard, A. D., Fisher, A. J., \& Nanthakumar, C. B. (2019). A high content, phenotypic 'scar-in-a-jar' assay for rapid quantification of collagen fibrillogenesis using disease-derived pulmonary fibroblasts. BMC Biomedical Engineering, 1(1), 14. https://doi.org/10.1186/s42490-019-0014-z

Guo, Y. C., \& Lu, L. G. (2020). Antihepatic Fibrosis Drugs in Clinical Trials. J Clin Trans/ Hepatol, 8(3), 304-312. https://doi.org/10.14218/JCTH.2020.00023

Hernandez-Gea, V., \& Friedman, S. L. (2011). Pathogenesis of liver fibrosis. Annu Rev Pathol, 6, 425-456. https://doi.org/10.1146/annurev-pathol-011110-130246

Huang, M., Liu, B., Liu, R., Li, J., Chen, J., Jiang, F., . . Liu, T. (2018). Aglycone Polyether Nanchangmycin and Its Homologues Exhibit Apoptotic and Antiproliferative Activities against Cancer Stem Cells. ACS Pharmacol Transl Sci, 1(2), 84-95. https://doi.org/10.1021/acsptsci.8b00007

Huczyński, A. (2012). Polyether ionophores-promising bioactive molecules for cancer therapy. Bioorg Med Chem Lett, 22(23), 7002-7010. https://doi.org/10.1016/j.bmcl.2012.09.046

Jones, K. T., \& Sharpe, G. R. (1994). Thapsigargin raises intracellular free calcium levels in human keratinocytes and inhibits the coordinated expression of differentiation markers. Exp Cell Res, 210(1), 71-76. https://doi.org/10.1006/excr.1994.1011

Karlsen, T. H., Folseraas, T., Thorburn, D., \& Vesterhus, M. (2017). Primary sclerosing cholangitis - a comprehensive review. J Hepatol, 67(6), 1298-1323. https://doi.org/10.1016/j.jhep.2017.07.022

Kaspar, J. W., \& Jaiswal, A. K. (2011). Tyrosine phosphorylation controls nuclear export of Fyn, allowing Nrf2 activation of cytoprotective gene expression. FASEB J, 25(3), 10761087. https://doi.org/10.1096/fj.10-171553

Kevin Ii, D. A., Meujo, D. A., \& Hamann, M. T. (2009). Polyether ionophores: broad-spectrum and promising biologically active molecules for the control of drug-resistant bacteria and parasites. Expert Opin Drug Discov, 4(2), 109-146. https://doi.org/10.1517/17460440802661443

Kisseleva, T., Cong, M., Paik, Y., Scholten, D., Jiang, C., Benner, C., . . Brenner, D. A. (2012). Myofibroblasts revert to an inactive phenotype during regression of liver fibrosis. Proc Natl Acad Sci U S A, 109(24), 9448-9453. https://doi.org/10.1073/pnas.1201840109 
Leite, S. B., Roosens, T., El Taghdouini, A., Mannaerts, I., Smout, A. J., Najimi, M., . . van Grunsven, L. A. (2016). Novel human hepatic organoid model enables testing of druginduced liver fibrosis in vitro. Biomaterials, 78, 1-10. https://doi.org/10.1016/j.biomaterials.2015.11.026

Li, J.-Q., Huang, M., Zhang, Y.-N., Liu, R., Zhang, Z.-R., Zhang, Q.-Y., . . . Liu, T. (2020). Aglycone polyether ionophores as broad-spectrum agents inhibit multiple enveloped viruses including SARS-CoV-2 in vitro and successfully cure JEV infected mice. bioRxiv, 2020.2010.2027.354563. https://doi.org/10.1101/2020.10.27.354563

Maher, J. J., \& McGuire, R. F. (1990). Extracellular matrix gene expression increases preferentially in rat lipocytes and sinusoidal endothelial cells during hepatic fibrosis in vivo. J Clin Invest, 86(5), 1641-1648. https://doi.org/10.1172/JCl114886

Matlock, M. K., Hughes, T. B., Dahlin, J. L., \& Swamidass, S. J. (2018). Modeling SmallMolecule Reactivity Identifies Promiscuous Bioactive Compounds. J Chem Inf Model, 58(8), 1483-1500. https://doi.org/10.1021/acs.jcim.8b00104

Maurer, B., Kollmann, S., Pickem, J., Hoelbl-Kovacic, A., \& Sexl, V. (2019). STAT5A and STAT5B-Twins with Different Personalities in Hematopoiesis and Leukemia. Cancers (Basel), 11(11). https://doi.org/10.3390/cancers11111726

Mederacke, I., Hsu, C. C., Troeger, J. S., Huebener, P., Mu, X., Dapito, D. H., . . Schwabe, R. F. (2013). Fate tracing reveals hepatic stellate cells as dominant contributors to liver fibrosis independent of its aetiology. Nat Commun, 4, 2823. https://doi.org/10.1038/ncomms3823

Mootha, V. K., Lindgren, C. M., Eriksson, K. F., Subramanian, A., Sihag, S., Lehar, J., . . . Groop, L. C. (2003). PGC-1alpha-responsive genes involved in oxidative phosphorylation are coordinately downregulated in human diabetes. Nat Genet, 34(3), 267-273. https://doi.org/10.1038/ng1180

Morgan, A. J., \& Jacob, R. (1994). Ionomycin enhances Ca2+ influx by stimulating storeregulated cation entry and not by a direct action at the plasma membrane. Biochem $\mathrm{J}$, 300 ( Pt 3), 665-672. https://doi.org/10.1042/bj3000665

Murphy, S. L., Xu, J., Kochanek, K. D., Arias, E., \& Tejada-Vera, B. (2021). Deaths: Final Data for 2018. Natl Vital Stat Rep, 69(13), 1-83.

Pagès, G., Lenormand, P., L'Allemain, G., Chambard, J. C., Meloche, S., \& Pouysségur, J. (1993). Mitogen-activated protein kinases p42mapk and p44mapk are required for fibroblast proliferation. Proc Natl Acad Sci U S A, 90(18), 8319-8323. https://doi.org/10.1073/pnas.90.18.8319

Pang, M., \& Zhuang, S. (2010). Histone deacetylase: a potential therapeutic target for fibrotic disorders. J Pharmacol Exp Ther, 335(2), 266-272. https://doi.org/10.1124/jpet.110.168385

Piñero, J., Ramírez-Anguita, J. M., Saüch-Pitarch, J., Ronzano, F., Centeno, E., Sanz, F., \& Furlong, L. I. (2020). The DisGeNET knowledge platform for disease genomics: 2019 update. Nucleic Acids Res, 48(D1), D845-D855. https://doi.org/10.1093/nar/gkz1021

Rausch, K., Hackett, B. A., Weinbren, N. L., Reeder, S. M., Sadovsky, Y., Hunter, C. A., .. . Cherry, S. (2017). Screening Bioactives Reveals Nanchangmycin as a Broad Spectrum Antiviral Active against Zika Virus. Cell Rep, 18(3), 804-815. https://doi.org/10.1016/j.celrep.2016.12.068

Ribeiro, D., Freitas, M., Rocha, S., Lima, J. L. F. C., Carvalho, F., \& Fernandes, E. (2018). Calcium Pathways in Human Neutrophils-The Extended Effects of Thapsigargin and ML-9. Cells, 7(11). https://doi.org/10.3390/cells7110204

Rusanescu, G., Qi, H., Thomas, S. M., Brugge, J. S., \& Halegoua, S. (1995). Calcium influx induces neurite growth through a Src-Ras signaling cassette. Neuron, 15(6), 14151425. https://doi.org/10.1016/0896-6273(95)90019-5

Sodhi, K., Srikanthan, K., Goguet-Rubio, P., Nichols, A., Mallick, A., Nawab, A., . . Shapiro, J. I. (2017). pNaKtide Attenuates Steatohepatitis and Atherosclerosis by Blocking $\mathrm{Na} / \mathrm{K}-\mathrm{ATP}$ ase/ROS Amplification in C57BI6 and ApoE Knockout Mice Fed a Western Diet. Sci Rep, 7(1), 193. https://doi.org/10.1038/s41598-017-00306-5 
Subramanian, A., Tamayo, P., Mootha, V. K., Mukherjee, S., Ebert, B. L., Gillette, M. A., . . Mesirov, J. P. (2005). Gene set enrichment analysis: a knowledge-based approach for interpreting genome-wide expression profiles. Proc Natl Acad Sci U S A, 102(43), 15545-15550. https://doi.org/10.1073/pnas.0506580102

Sun, Y., Zhou, X., Liu, J., Bao, K., Zhang, G., Tu, G., . . . Deng, Z. (2002). 'Streptomyces nanchangensis', a producer of the insecticidal polyether antibiotic nanchangmycin and the antiparasitic macrolide meilingmycin, contains multiple polyketide gene clusters. Microbiology (Reading), 148(Pt 2), 361-371. https://doi.org/10.1099/00221287-148-2$\underline{361}$

Sun, Y. M., Chen, S. Y., \& You, H. (2020). Regression of liver fibrosis: evidence and challenges. Chin Med J (Engl), 133(14), 1696-1702. https://doi.org/10.1097/CM9.0000000000000835

Svenningsen, E. B., Thyrsted, J., Blay-Cadanet, J., Liu, H., Lin, S., Villameriel, J. M., .. . Poulsen, T. B. (2020). lonophore antibiotic X-206 is a potent and selective inhibitor of SARS-CoV-2 infection <em>in vitro</em>. bioRxiv, 2020.2006.2014.149153. https://doi.org/10.1101/2020.06.14.149153

Tian, J., \& Xie, Z. J. (2008). The Na-K-ATPase and calcium-signaling microdomains. Physiology (Bethesda), 23, 205-211. https://doi.org/10.1152/physiol.00008.2008

Troeger, J. S., Mederacke, I., Gwak, G. Y., Dapito, D. H., Mu, X., Hsu, C. C., . . Schwabe, R. F. (2012). Deactivation of hepatic stellate cells during liver fibrosis resolution in mice. Gastroenterology, $143(4)$, https://doi.org/10.1053/j.gastro.2012.06.036 1073-1083.e1022.

Xia, Z., Dudek, H., Miranti, C. K., \& Greenberg, M. E. (1996). Calcium influx via the NMDA receptor induces immediate early gene transcription by a MAP kinase/ERK-dependent mechanism. J Neurosci, 16(17), 5425-5436.

Xu, Y., Sun, T., Zeng, K., Xu, M., Chen, J., Xu, X., . . . Mao, X. (2020). Anti-bacterial and antiviral nanchangmycin displays anti-myeloma activity by targeting Otub1 and c-Maf. Cell Death Dis, 11(9), 818. https://doi.org/10.1038/s41419-020-03017-4

Yoon, S., Kang, G., \& Eom, G. H. (2019). HDAC Inhibitors: Therapeutic Potential in FibrosisAssociated Human Diseases. Int $J$ Mol Sci, 20(6). https://doi.org/10.3390/ijms20061329

Younossi, Z. M., Koenig, A. B., Abdelatif, D., Fazel, Y., Henry, L., \& Wymer, M. (2016). Global epidemiology of nonalcoholic fatty liver disease-Meta-analytic assessment of prevalence, incidence, and outcomes. Hepatology, 64(1), 73-84. https://doi.org/10.1002/hep.28431

Zhang, D. Y., Goossens, N., Guo, J., Tsai, M. C., Chou, H. I., Altunkaynak, C., . . Friedman, S. L. (2016). A hepatic stellate cell gene expression signature associated with outcomes in hepatitis $\mathrm{C}$ cirrhosis and hepatocellular carcinoma after curative resection. Gut, 65(10), 1754-1764. https://doi.org/10.1136/gutjnl-2015-309655

Zhao, X. K., Cheng, Y., Liang Cheng, M., Yu, L., Mu, M., Li, H., . . Zhang, Q. (2016). Focal Adhesion Kinase Regulates Fibroblast Migration via Integrin beta-1 and Plays a Central Role in Fibrosis. Sci Rep, 6, 19276. https://doi.org/10.1038/srep19276

Zhao, X. K., Yu, L., Cheng, M. L., Che, P., Lu, Y. Y., Zhang, Q., . . Ding, Q. (2017). Focal Adhesion Kinase Regulates Hepatic Stellate Cell Activation and Liver Fibrosis. Sci Rep, 7(1), 4032. https://doi.org/10.1038/s41598-017-04317-0

Zhou, J., Yi, Q., \& Tang, L. (2019). The roles of nuclear focal adhesion kinase (FAK) on Cancer: a focused review. J Exp Clin Cancer Res, 38(1), 250. https://doi.org/10.1186/s13046$\underline{019-1265-1}$ 
Figures and Legends
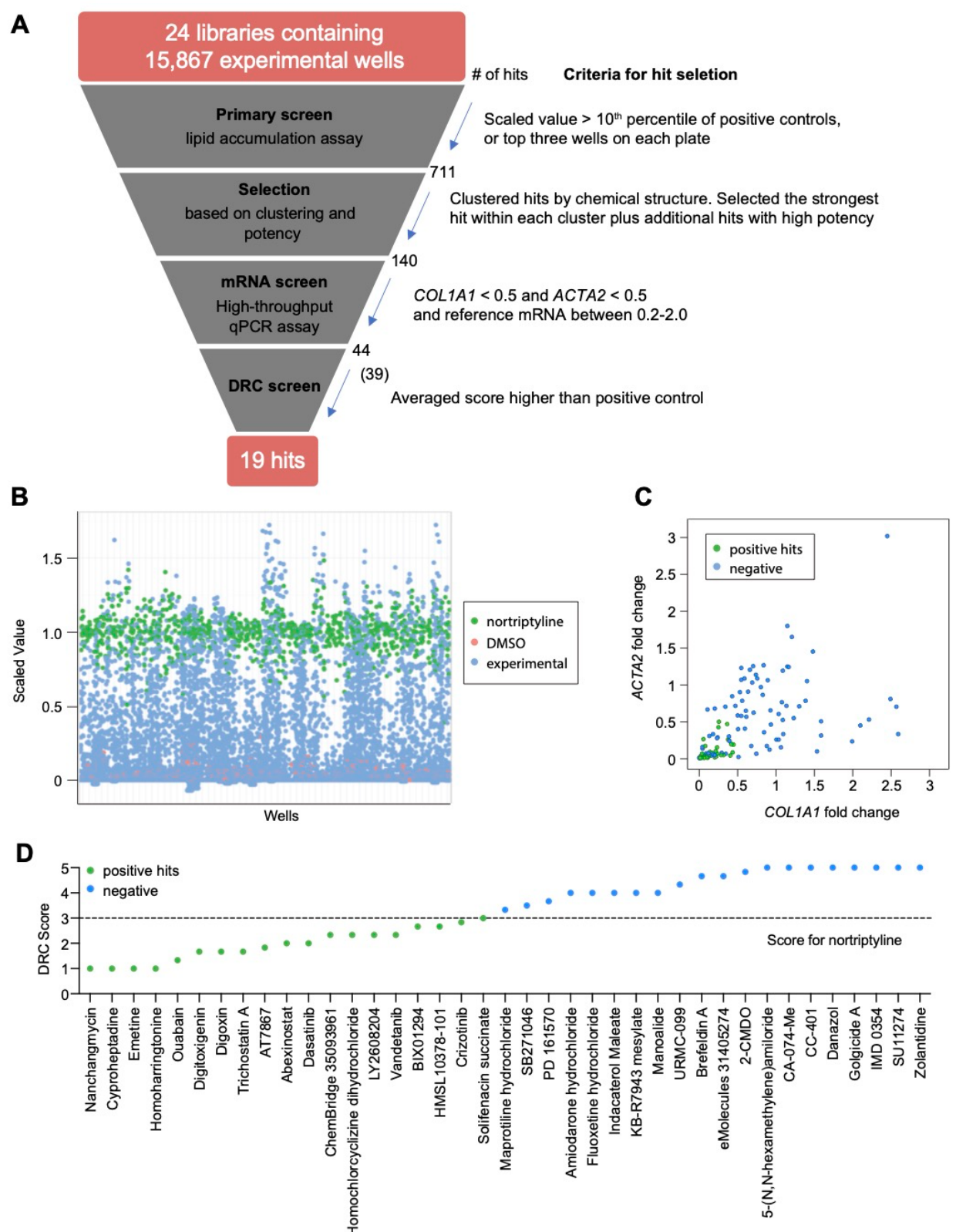
indicated at each step. The number in parenthesis represents the number of compounds tested in the final dose response curve (DRC) analysis due to availability. B. Results of the primary lipid accumulation screen. Each dot indicates the mean scaled value of two replicates for each condition at 48 hours. Red dots represent negative control wells (DMSO), green dots represent positive control wells (nortriptyline, $27 \mu \mathrm{M}$ ), and blue dots represent experimental wells. C. Results of the secondary mRNA screen. Each dot indicates the mean fold change of ACTA2 and COL1A1 after treatment with compounds (normalized to DMSO controls). PSMB2 was used as the reference gene $(n=4)$. Green dots represent positive hits $(<0.5)$, and blue dots represent non-hits (negative). D. Dose-response curves were plotted for 39 compounds and were scored by three researchers independently. The mean score for each compound was plotted. The dotted line indicates the score of the positive control nortriptyline. Green dots represent positive hits, and blue dots represent non-hits (negative). This figure has two supplements. 
A
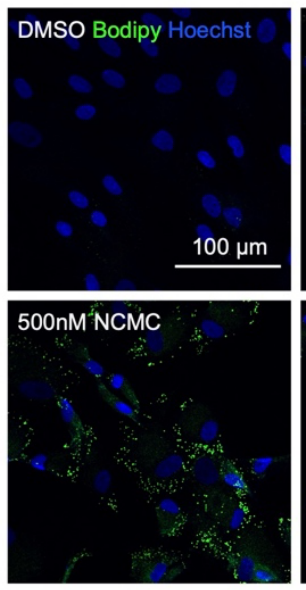

C

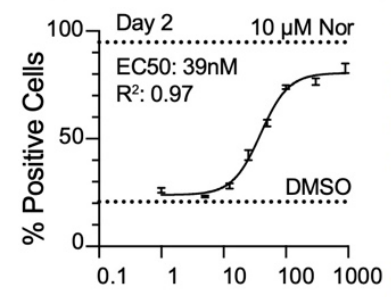

NCMC concentration (nM)

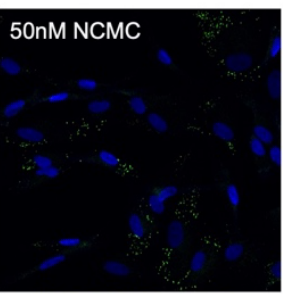

B

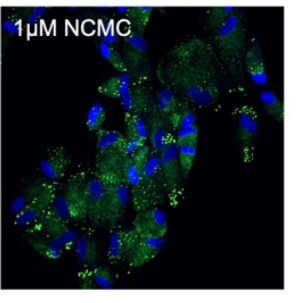

D

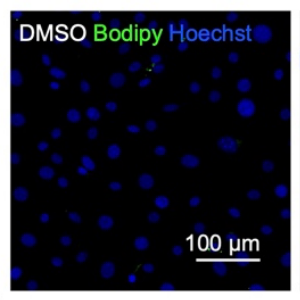

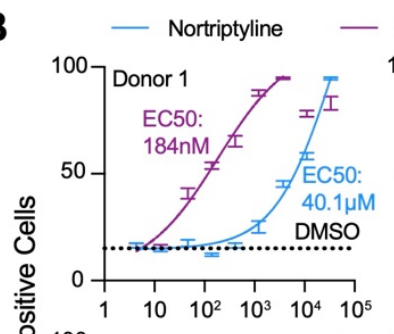
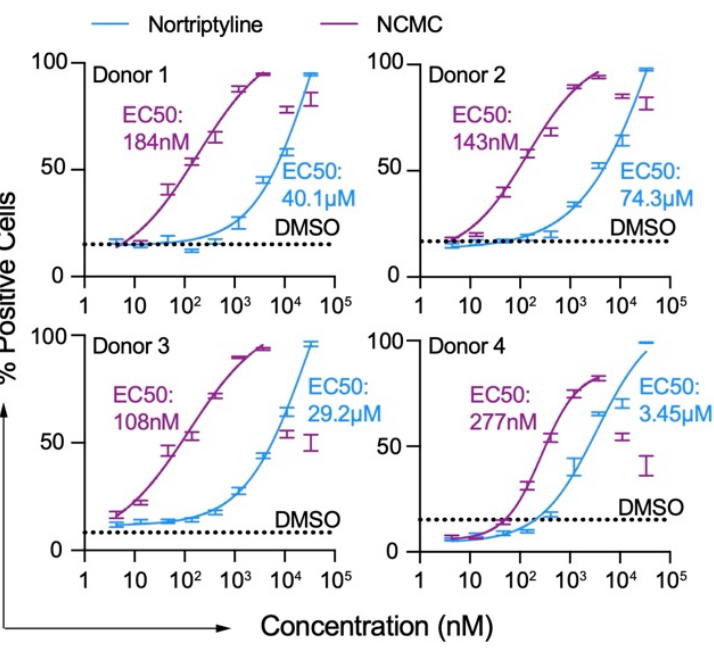

$\rightarrow$ Concentration $(\mathrm{nM})$

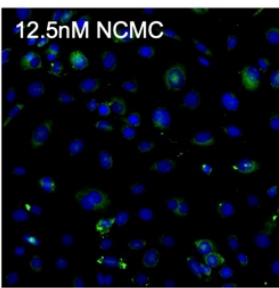

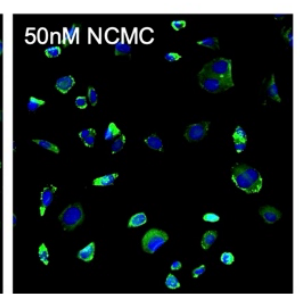

Figure 2. NCMC induces lipid accumulation in HSCs

A. Representative microscopic images of HSCs treated with DMSO and NCMC for 48 hours. Cells were stained with Bodipy to identify lipid droplets (green) and Hoechst to define nuclei (blue). Scale bar represents $100 \mu \mathrm{m}$. B. Dose response curves for NCMC (purple) and nortriptyline (blue) in HSCs isolated from four different human donors at 48 hours. Dotted line represents the mean percentage of Bodipy-positive cells in DMSO control wells. Error bars represent mean \pm SEM $(n=12)$. One experiment was performed for each of four donor lines. Curves were generated by fitting the data to a sigmoidal model. The data from the highest two concentrations of NCMC treatment $(11$ and $33 \mu \mathrm{M})$ were not used for fitting due to higher toxicity at these concentrations, as indicated by cell number (Figure 2 - figure supplement 1 ). C. Dose response curve for NCMC treatment in murine primary HSCs at 48 hours. Dotted lines represent the averaged percentage of Bodipy-positive cells in DMSO negative control wells (lower) and nortriptyline positive control wells (10 $\mu \mathrm{M}$, upper). Error bars represent mean \pm SEM $(n=6)$. D. Representative images of murine HSCs treated with DMSO and NCMC for 48 hours and stained with Bodipy to identify lipid droplets (green) and Hoechst to define nuclei (blue). Scale bar represents $100 \mu \mathrm{m}$. This figure has two supplements. 
A

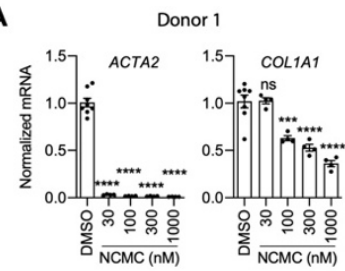

C
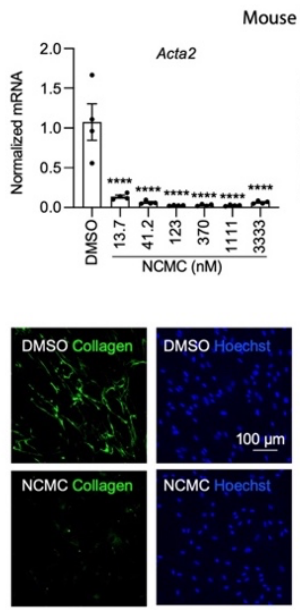

G

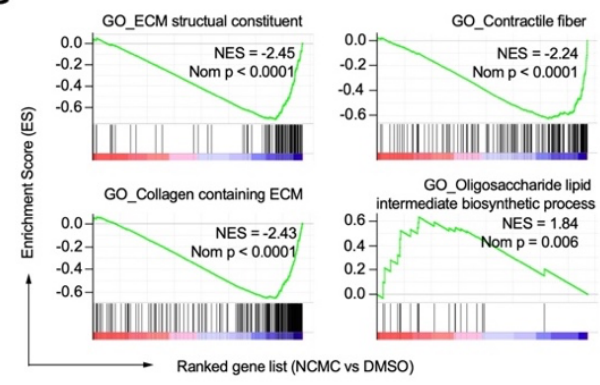

$\mathbf{F}$
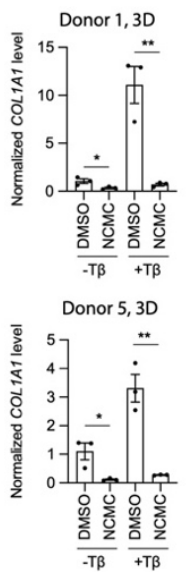

H
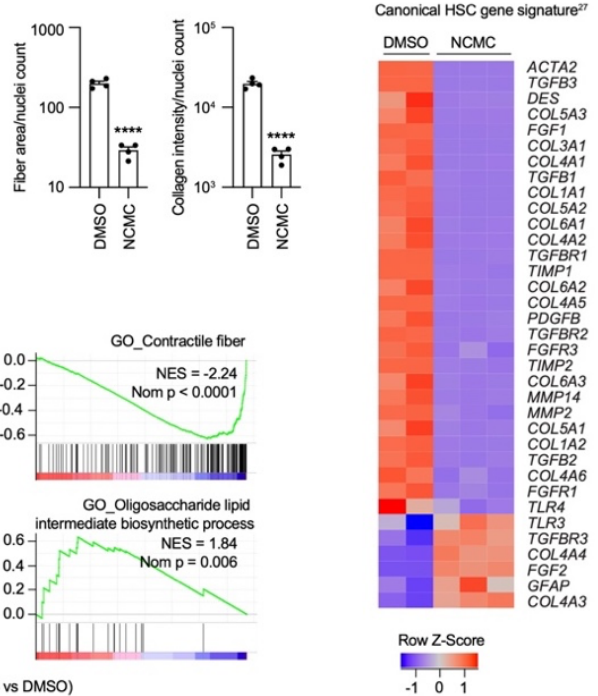

Figure 3. NCMC inhibits expression of fibrotic genes in HSCs

A-B. Effect of $48 \mathrm{hr}$ NCMC treatment on ACTA2 and COL1A1 in HSCs from human donors 1 (A) and 3 (B). Error bars represent mean \pm SEM $(n=3)$. Data are representative of three independent experiments. ns indicates not significant, ${ }^{* *}$ indicates $p<0.001$, and $* * * *$ indicates $p<0.0001$ (one-way ANOVA test). C. Effect of 48-hour NCMC treatment on Acta2 and Col1a1 in primary mouse HSCs. Error bars represent mean \pm SEM $(n=4)$. Data are representative of three independent experiments. ${ }^{* * * *}$ indicates $p<0.0001$ (one-way ANOVA test). D-E. Effect of $48 \mathrm{hr}$ NCMC treatment $(1 \mu \mathrm{M})$ on collagen deposition in ECM. D: representative images. Scale bar represents $100 \mu \mathrm{m}$. Collagen protein is indicated in green and nuclei for the same field are indicated in blue. E: quantified results. Error bars represent mean \pm SEM $(n=4)$. Data are representative of three independent experiments. ${ }^{* * *}$ indicates $p<0.0001$ (Student's t-test). F. qPCR analysis of COL1A1 in HSC-hepatocyte spheroids treated with NCMC with and without TGF- $\beta(T \beta)$. Error bars represent mean \pm SEM $(n=3)$. One experiment was performed for each donor shown. * indicates $p<0.05$ (Student's t-test) and ${ }^{* *}$ indicates $p<0.01$ (Student's t-test). Analysis was performed on day 3 (3D). G-H. RNA sequencing analysis of HSCs (donor 1 ) treated with DMSO or $1 \mu \mathrm{M}$ NCMC for 48 hours. G: Representative gene sets from the gene set enrichment analysis (GSEA). NES refers to normalized enrichment score. Nom P refers to Nominal $P$ value. Vertical black lines refer to affected genes in the indicated signatures. Red color indicates positive correlation, and blue color indicates negative correlation. H: Heatmap showing RNA-seq expression for the canonical HSC gene signature (Zhang et al., 2016). All genes from the signature that are expressed in HSCs (with a minimum of five reads) were shown regardless of their expression patterns. Z-score values are also provided in Supplementary Table 8. This figure has one supplement. 

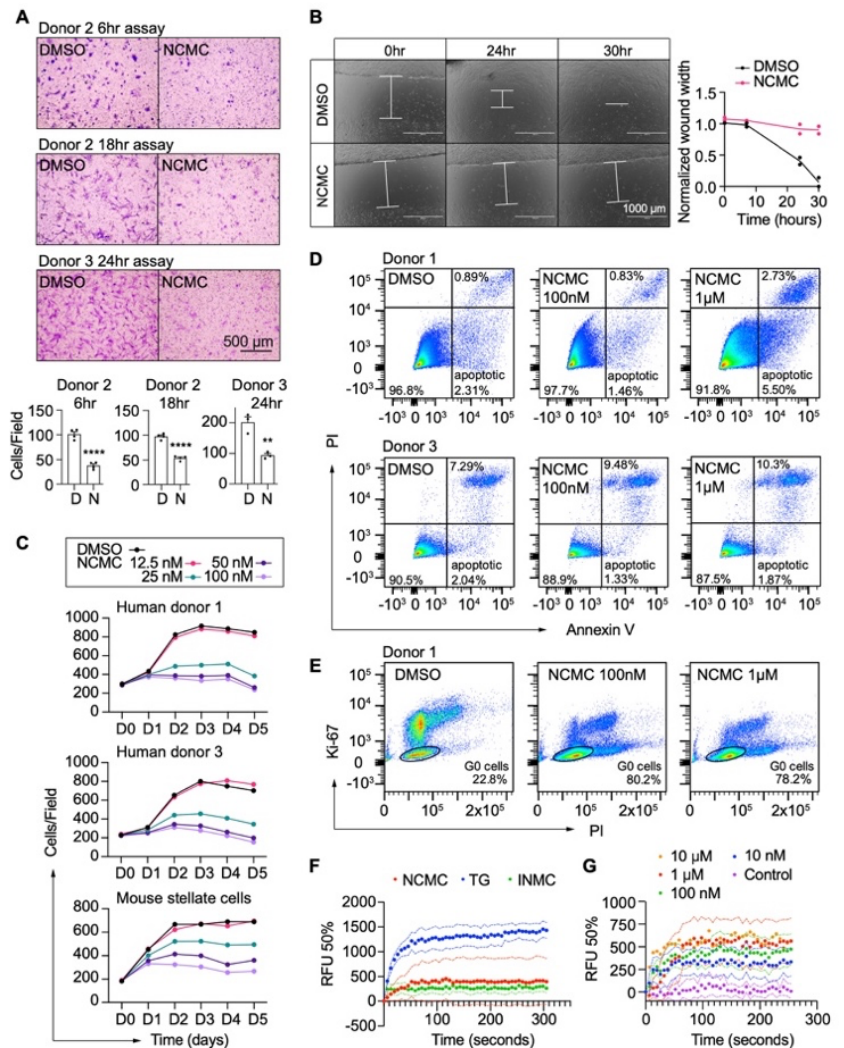

Figure 4. NCMC inhibits HSC migration and proliferation and increases cytosolic calcium concentration

A. Transwell migration assay results of HSCs treated with DMSO or $1 \mu \mathrm{M} \mathrm{NCMC} \mathrm{for} 48 \mathrm{hr}$. Top: representative images. Scale bar represents $500 \mu \mathrm{m}$. Bottom: quantification of migrated cells ( $n=3$ for each experiment). ${ }^{* *}$ indicates $p<0.01$, and ${ }^{* * * *}$ indicates $p<0.0001$ (Student's t-test). B. Wound healing assay results of HSCs treated with DMSO or NCMC. HSCs were seeded in complete medium, and immediately after generating the wound field, DMSO and 1 $\mu \mathrm{M} N C M C$ were added. The closure of the wound field was monitored for up to 30 hours as indicated. Left: representative images. White bars highlight the width of the wound field. Scale bar represents $1000 \mu \mathrm{m}$. Right: quantification of wound width. $(n=2)$. Data are representative of three independent experiments. C. Cell count for HSCs treated with DMSO or NCMC over the indicated time in days. Top: human HSCs from donor 1. Middle: human HSCs from donor 3. Bottom: mouse HSCs. Error bars represent mean \pm SEM $(n=6)$ but are too small to be visualized. One experiment was performed for each HSC line shown. D. Flow cytometry analysis of Annexin V and propidium iodide (PI) stained HSCs from human donor 1 (top) and 3 (bottom) treated with DMSO or NCMC for 48 hours. Plots are representative of two independent experiments. E. Flow cytometry analysis of Ki-67 and PI stained HSCs from human donor 1 treated with DMSO or NCMC for 24 hours. Plots are representative of two independent experiments. F. Measurement of cytosolic calcium level using fluo-4 NW. HSCs from donor 3 were pre-loaded with fluo-4 NW and fluorescent intensity was read immediately after adding compounds (NCMC: nanchangmycin (red), TG: thapsigargin (blue), INMC: ionomycin (green)). The plot demonstrates results from three independent experiments. Solid dots represent mean, and dotted lines represent SEM ( $n=3)$. RFU: Relative fluorescence unit. G. Measurement of cytosolic calcium level after adding NCMC at indicated concentrations in HSCs from donor 3 (magenta: control (no compound, no DMSO), blue: $10 \mathrm{nM}$, green: $100 \mathrm{nM}$, red $1 \mu \mathrm{M}$, orange: $10 \mu \mathrm{M}$ ). The plot demonstrates results from one experiment for $10 \mu \mathrm{M}$ and five independent experiments for the other concentrations. Solid dots represent mean, and dotted lines represent SEM $(n=3)$. This figure has two supplements. 
A

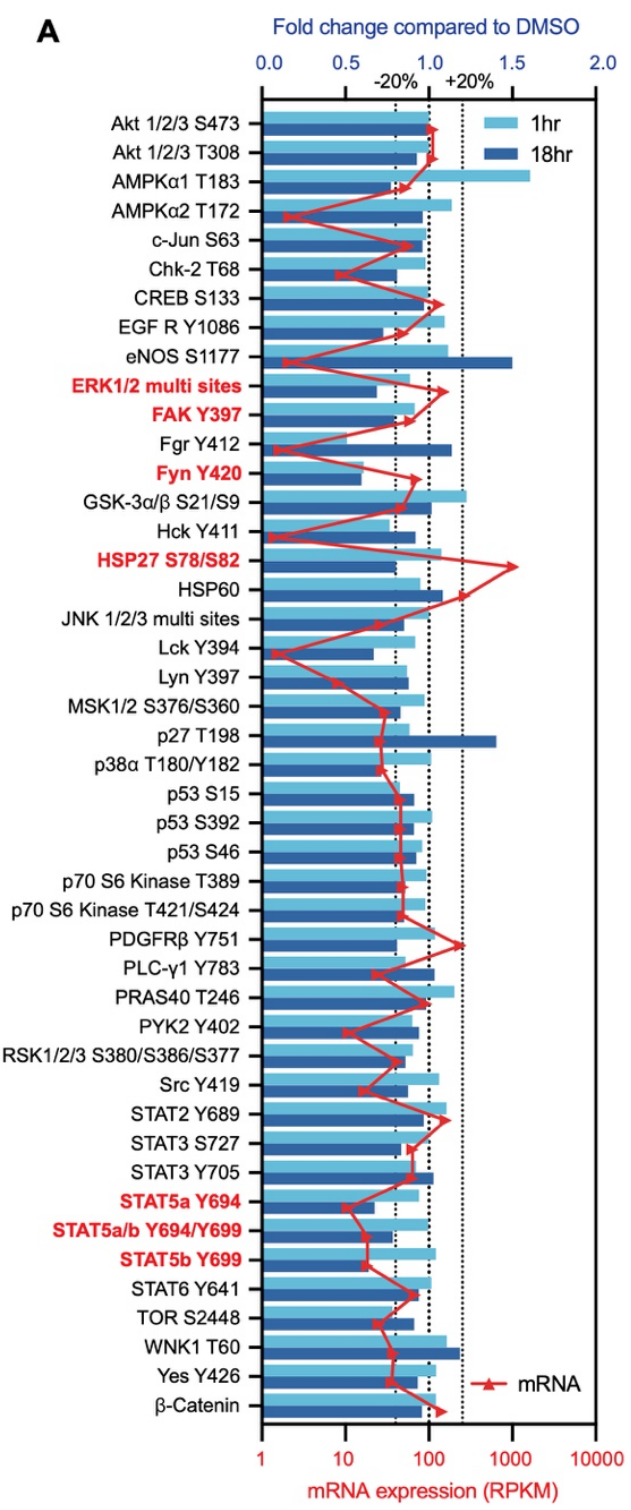

B
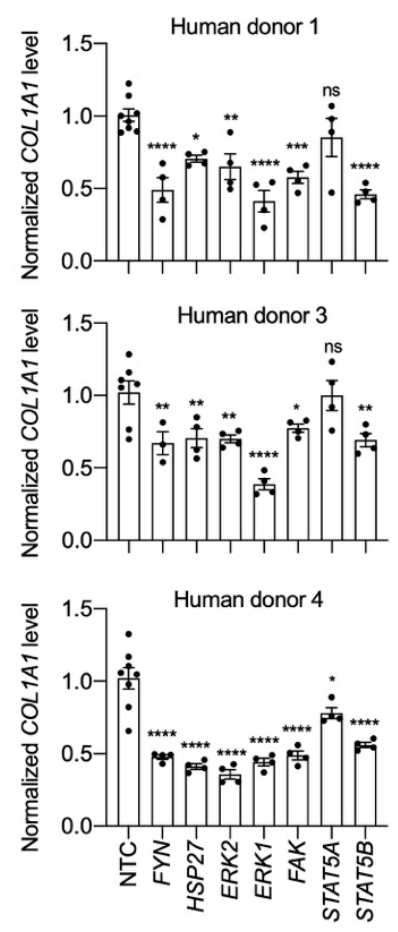

C
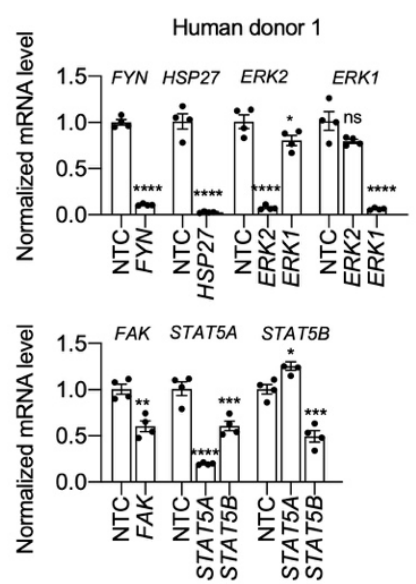

Figure 5. Multiple kinases mediate the effect of NCMC on COL1A1 expression

A. Kinase array analysis of HSCs treated with DMSO or $1 \mu \mathrm{M} \mathrm{NCMC}$ for 1 or 18 hours. Blue bars indicate mean fold change $(n=2)$ in phosphorylation at specified sites in NCMC-treated cells compared to DMSO-treated cells at 1 hour (light blue) or 18 hours (dark blue). Red triangles indicate the mean RPKM of each corresponding kinase mRNA based on RNA sequencing of HSCs (Chen et al., 2017). The three dotted lines represent 20\% increase/decrease or no change in phosphorylation. Kinases highlighted in red were chosen for further investigation. B. The expression of each candidate kinase gene was depleted using pooled siRNAs, and after 72 hours, COL1A1 level was determined by qRT-PCR in HSCs isolated from human donor 1 (top), 3 (middle), or 4 (bottom). A non-targeting siRNA is used as a control (NTC). Error bars represent mean \pm SEM $(n \geq 4$, as indicated by the number of dots). ns indicates not significant $(p>0.05),{ }^{*}$ indicates $p<0.05$, ${ }^{* *}$ indicates $p<0.01,{ }^{* * *}$ indicates $p<0.001$, and ${ }^{* * * *}$ indicates $p<0.0001$ (one-way ANOVA test). C. Knockdown efficiency of each siRNA pool in HSCs from human donor 1. Error bars represent mean \pm SEM $(n \geq 4)$. ns indicates not significant $(p>0.05)$, ${ }^{*}$ indicates $p<0.05$, ${ }^{* *}$ indicates $p<0.01$, ${ }^{* * *}$ indicates $p<0.001$, and ${ }^{* * *}$ indicates $p<0.0001$ (Student's t-test performed for $F Y N, H S P 27$, and FAK depletion, and one-way ANOVA test performed for ERK1, ERK2, STAT5A and STAT5B depletion). This figure has one supplement. 


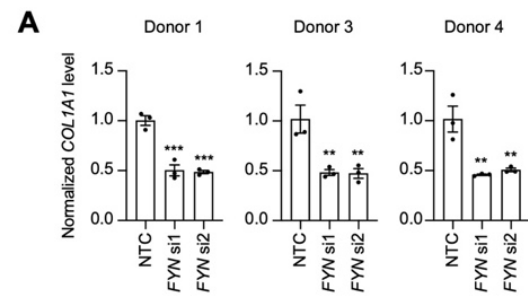

B
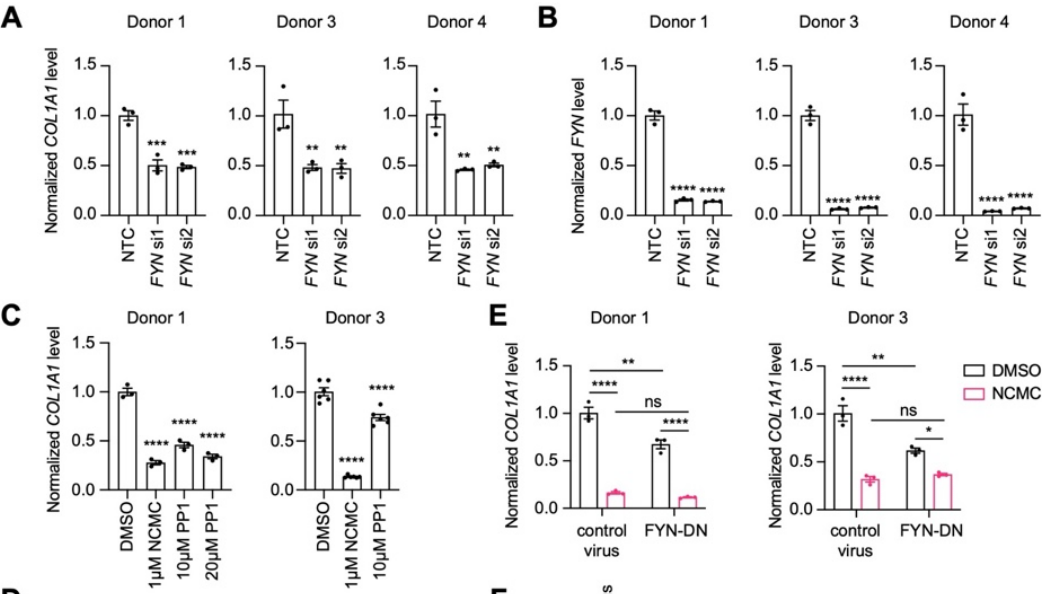

Donor 3
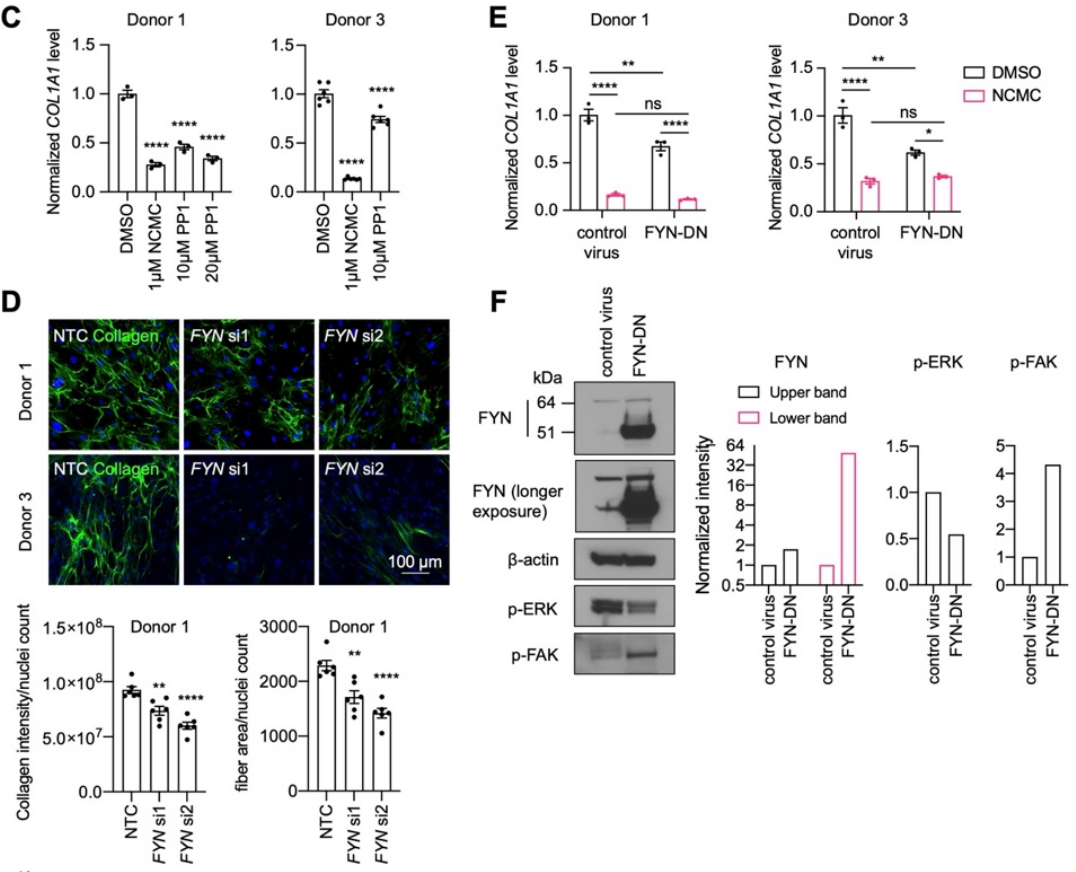

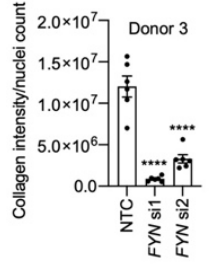

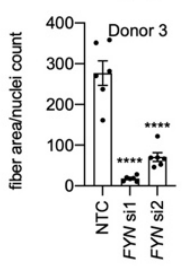

Figure 6. FYN/ERK pathway regulates collagen expression

A-B. FYN was depleted in HSCs with two siRNAs (si1 and si2). The expression levels of COL1A1 (A) and FYN (B) were analyzed by qPCR after 72 hours in comparison to a nontargeting control siRNA (NTC). Error bars represent mean \pm SEM $(n=3)$ for each of three donor lines. ${ }^{* *}$ indicates $p<0.01,{ }^{* * *}$ indicates $p<0.001$, and ${ }^{* * * *}$ indicates $p<0.0001$ (one-way ANOVA test). C. HSCs were treated with NCMC or 1-Naphthyl PP1 (PP1) for 48 hours. COL1A1 level was analyzed by qPCR. Error bars represent mean \pm SEM ( $n=3$ for donor 1 , and $n=6$ for donor 3 ). Data are representative of three independent experiments for donor 1 and experiment for donor $3 .{ }^{* * * *}$ indicates $p<0.0001$ (one-way ANOVA test). D. Effect of FYNdepletion on collagen deposition in ECM. Top: representative images. Scale bar represents $100 \mu \mathrm{m}$. Bottom: quantified results. Error bars represent mean $\pm \operatorname{SEM}(n=6)$. Data are representative of two independent experiments. ${ }^{* *}$ indicates $p<0.01$, and ${ }^{* * * *}$ indicates $p<$ 0.0001 (one-way ANOVA test). E. HSCs transduced with control virus or virus containing the cDNA encoding dominant negative mutant FYN (FYN-DN) were treated with DMSO or 100 $\mathrm{nM} \mathrm{NCMC}$ for 48 hours. Expression of COL1A1 was quantified by qPCR. Error bars represent mean \pm SEM $(n=3)$. Data are representative of three independent experiments). ns indicates not significant $(p>0.05),{ }^{*}$ indicates $p<0.05,{ }^{* *}$ indicates $p<0.01$, and ${ }^{* * * *}$ indicates $p<$ 0.0001 (two-way ANOVA test). F. Phospho-ERK and phospho-FAK levels were determined by Western blot in control HSCs and HSCs overexpressing DN-FYN. Left: representative Western blot results. Right: quantified results. Representative of two independent experiments. This figure has four supplements. 

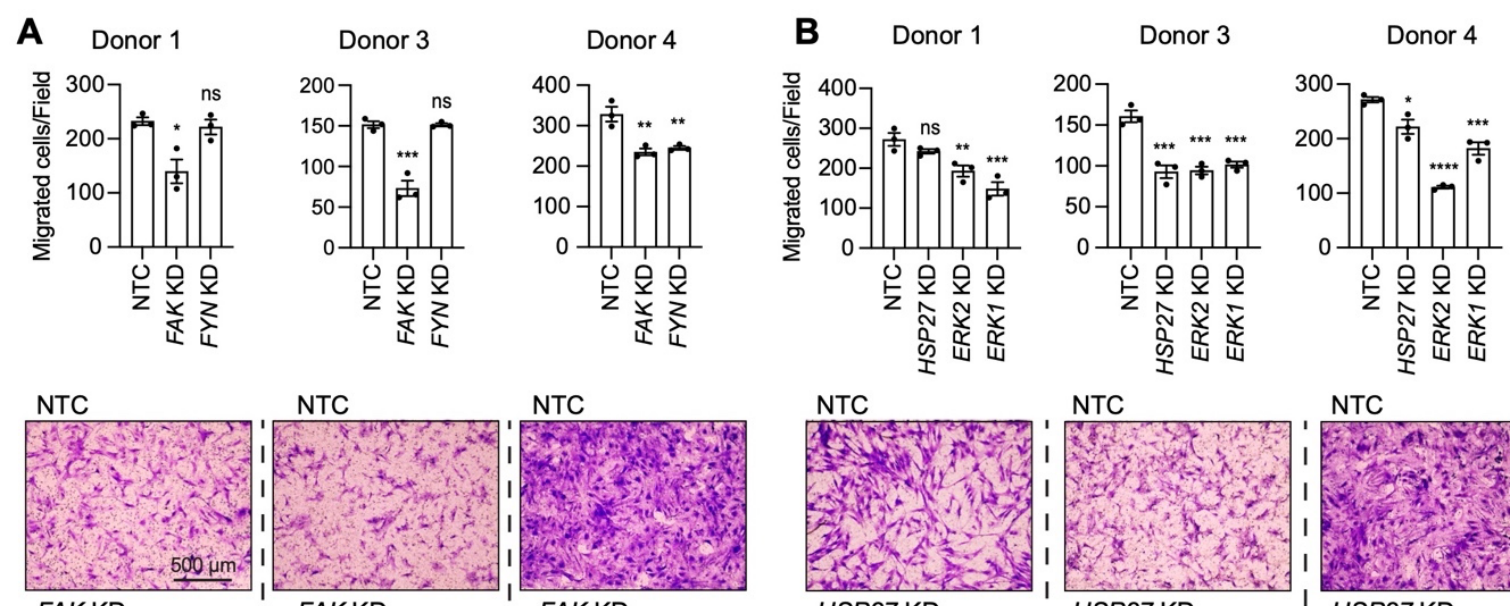

NTC
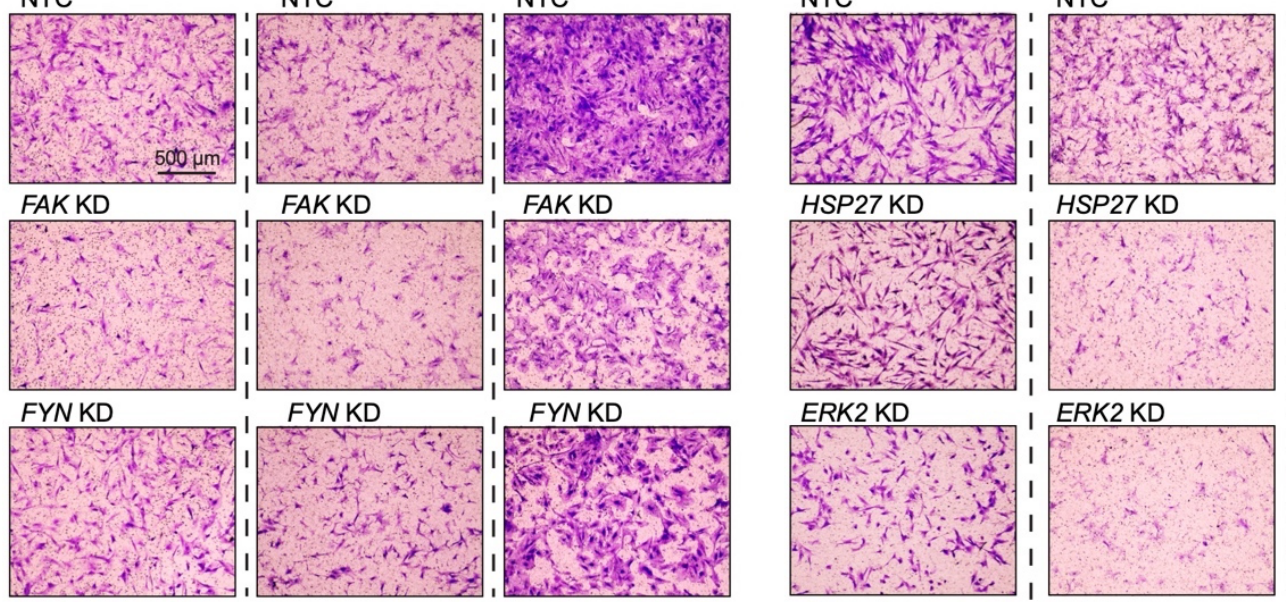

NTC
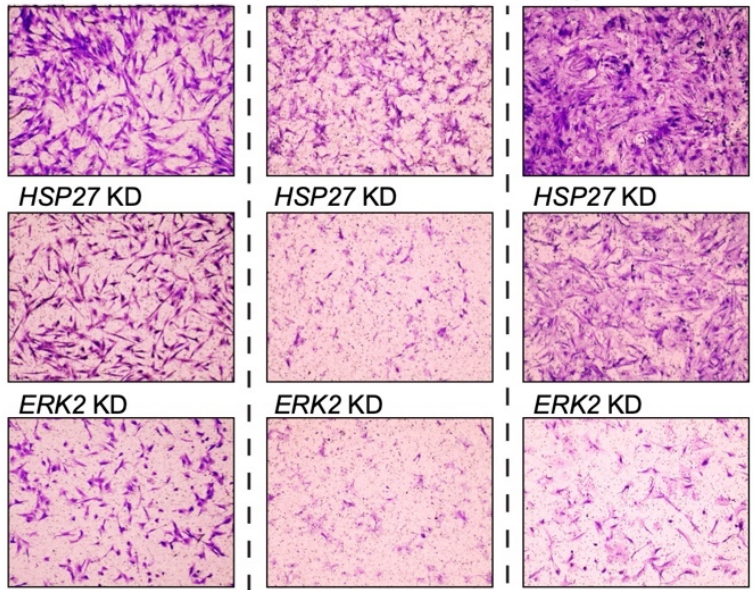

Chronic liver injury

Activated HSC
myofibroblast
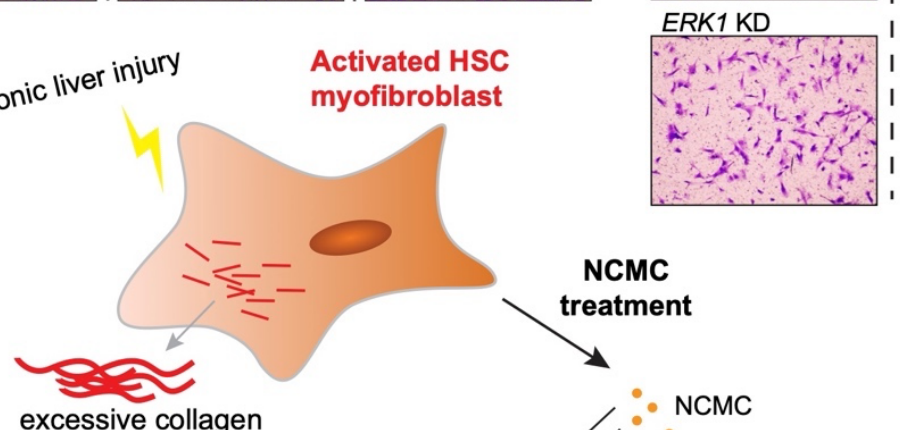

ERK2 KD

HSP27 KD

excessive collagen

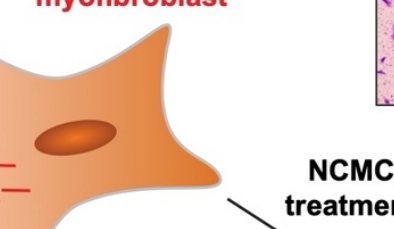

NCMC

Figure 7. ERK and FAK regulate HSC migration

A-B. Kinases were depleted in HSCs with pooled siRNAs. NTC indicates non-targeting siRNA control. KD indicates the gene transcript that is knocked down. After 72 hours, cells were seeded for transwell migration assay. Migration was assayed at 24 hours for HSCs from donor 1 and 4, and 6 hours for HSCs from donor 3. Top: quantified results. Error bars represent mean \pm SEM $(n=3)$. Results are shown for three donor lines. ns indicates not significant $(p>$ $0.05)$, ${ }^{*}$ indicates $p<0.05$, ${ }^{* *}$ indicates $p<0.01$, ${ }^{* * *}$ indicates $p<0.001$, and ${ }^{* * * *}$ indicates $p<$ 0.0001 (one-way ANOVA test). Bottom: representative images. Scale bar represents $500 \mu \mathrm{m}$. C. Schematic summarizing the signaling pathways triggered by NCMC treatment and the effect on HSC activity. Red lines represent collagen, orange spots represent NCMC, and green spots represent lipid droplets. 
Table 1. Candidates from small molecule screening

\begin{tabular}{ccc}
\hline Compound Name & DRC Score & Known function \\
\hline Cyproheptadine $^{*}$ & 1.0 & serotonin antagonist and antihistamine \\
Emetine $^{*}$ & 1.0 & anti-protozoal, inhibitor of Zika and Ebola viruses \\
Homoharringtonine $^{*}$ & 1.0 & translation elongation inhibitor \\
Nanchangmycin $^{*}$ & 1.0 & polyether ionophore antibiotic, inhibitor of Zika virus \\
Ouabain $^{*}$ & 1.3 & Na/K-ATPase inhibitor \\
Digitoxigenin $^{*}$ & 1.7 & Na/K-ATPase inhibitor \\
Digoxin * $^{*}$ & 1.7 & Na/K-ATPase inhibitor \\
Trichostatin A & 1.7 & histone deacetylase inhibitor \\
AT7867, HMSL10154-101-1 & 1.8 & multi-kinase inhibitor \\
PCI-24781 (Abexinostat) & 2.0 & histone deacetylase inhibitor \\
Dasatinib & 2.0 & multi-kinase inhibitor \\
ChemBridge 35093961 & 2.3 & IKK inhibitor \\
Homochlorcyclizine dihydrochloride & 2.3 & antihistamine \\
LY2608204 & 2.3 & glucokinase activator \\
Vandetanib & 2.3 & multi-kinase inhibitor \\
BIX01294 (hydrochloride hydrate) & 2.7 & G9a histone methyltransferase inhibitor \\
HMSL10378-101 & 2.7 & predicted to target GSK3B at 1 nM (ChEMBL) \\
Crizotinib & 2.8 & multi-kinase inhibitor \\
Solifenacin succinate & 3.0 & muscarinic receptor antagonist \\
\hline
\end{tabular}




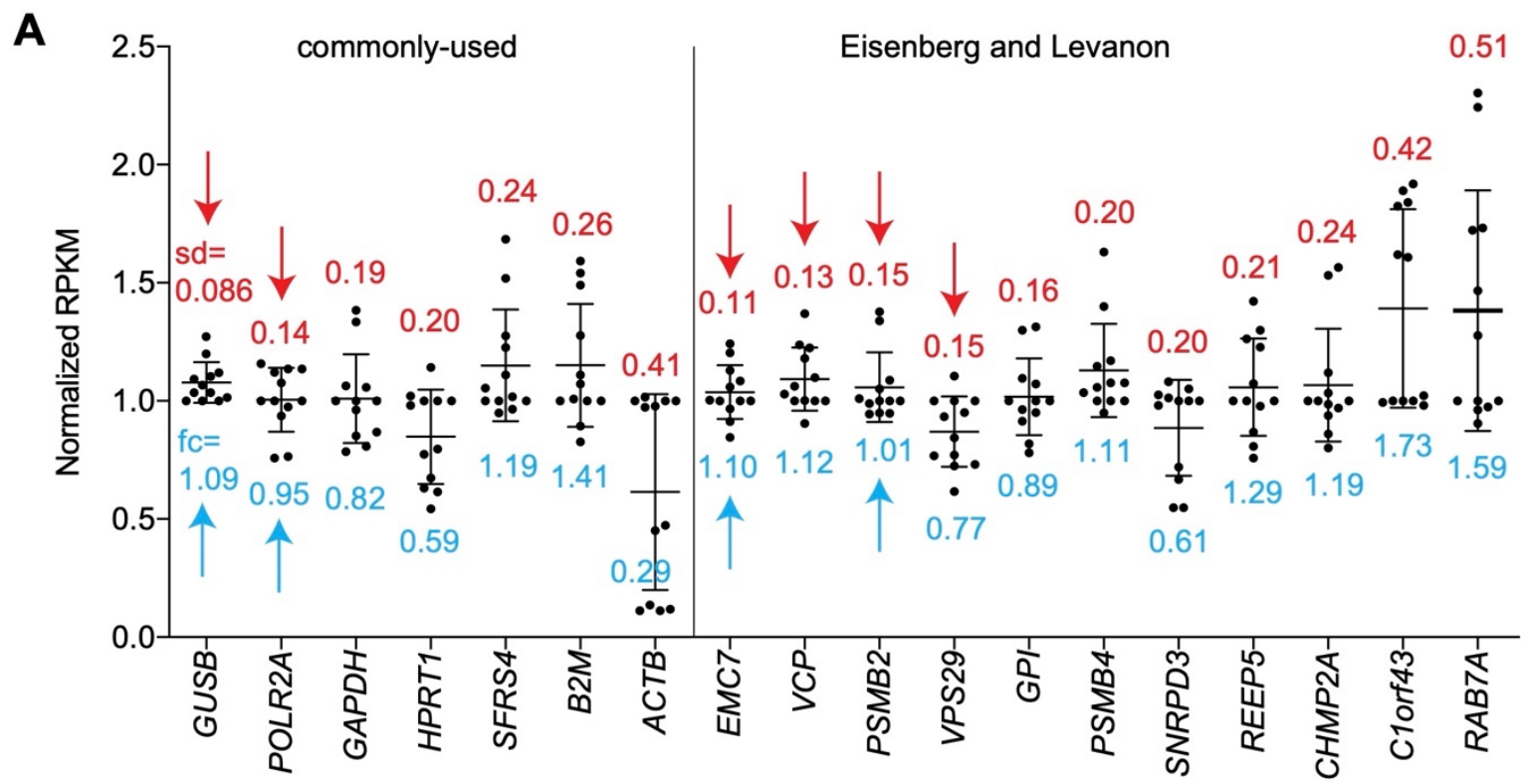

B

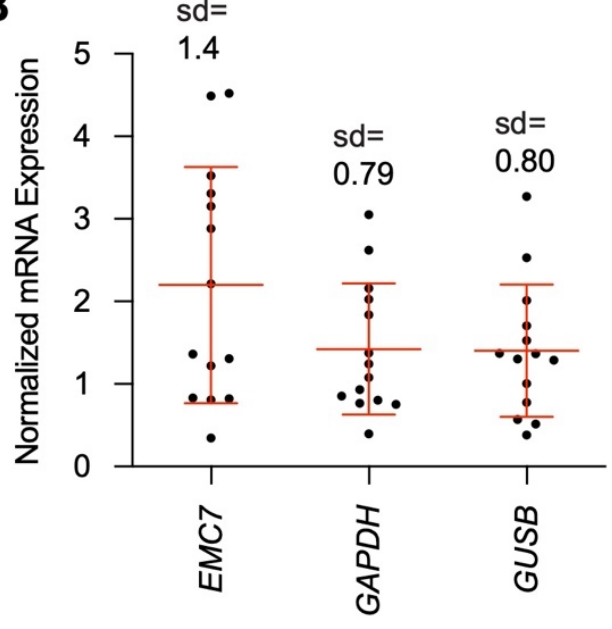

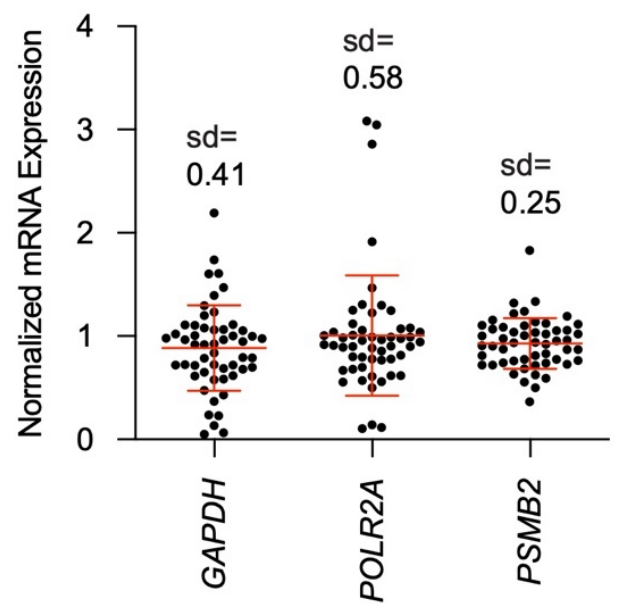

1203

Figure 1 - figure supplement 1 . Selection of PSMB2 as the reference mRNA for the qPCR-based secondary screening assay

A. Expression of house-keeping genes in HSCs was plotted by RPKM as previously quantified (Chen et al., 2017). Three pairs of samples were used in this analysis: ceramide treatment vs control, nortriptyline treatment vs control, nortriptyline treatment vs control in TGF- $\beta$ treated HSCs (Chen et al., 2017). Each dot represents the result from one sample, and bars represent mean \pm standard deviation (sd). The value of sd is indicated above each mRNA. The fold change (fc) of EMC7, VCP, PSMB2 and VPS29 in inactivated HSCs (ceramide or nortriptylinetreated) compared to corresponding control groups is indicated below the dots. Two groups of reference mRNAs were analyzed -- commonly used ones (left) and genes that are expressed uniformly across tissues (Eisenberg \& Levanon, 2013). Red arrows indicate samples with sd of 0.15 or less. Blue arrows highlight the reference mRNAs with fold change of no more than $10 \%$ in inactivated HSCs and were selected for further analysis. GAPDH, which is used routinely as a reference control was also included. B. Quantification of expression of $E M C 7$, GAPDH, GUSB, POLR2A, and PSMB2 from HSC cDNA samples (left: $\mathrm{n}=14$, right: $\mathrm{n}=53$ ) that were reverse transcribed from equal amounts of total input RNA. All samples were normalized to the mean value of its own control group before they were combined for each of the reference mRNAs. Each dot represents the result from one sample, and bars represent mean \pm standard deviation (sd) of all the tested samples. The value of sd is indicated above each mRNA. 

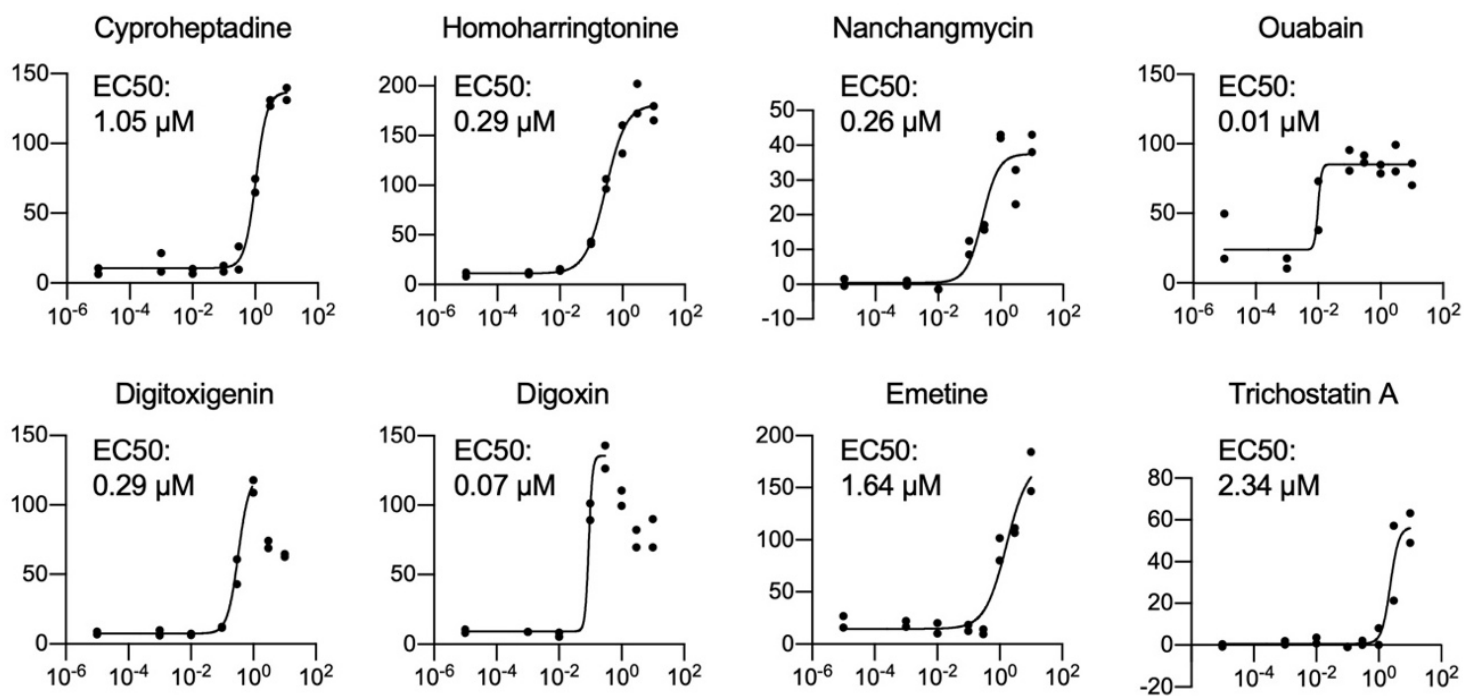

Trichostatin A

Abexinostat
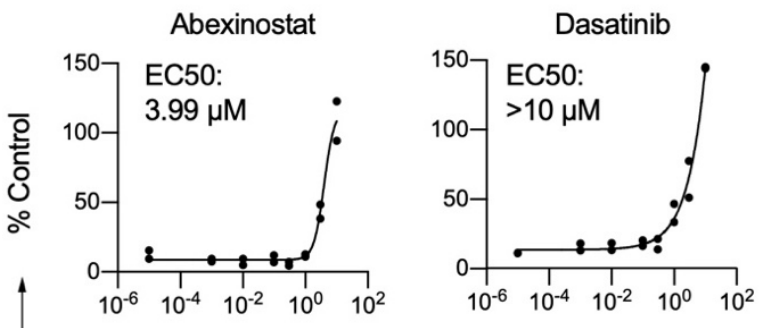

ChemBridge 35093961

EC50:

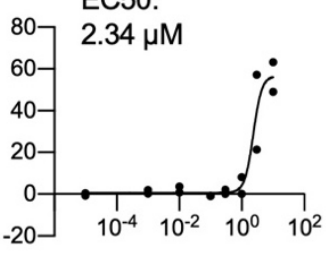

AT7867
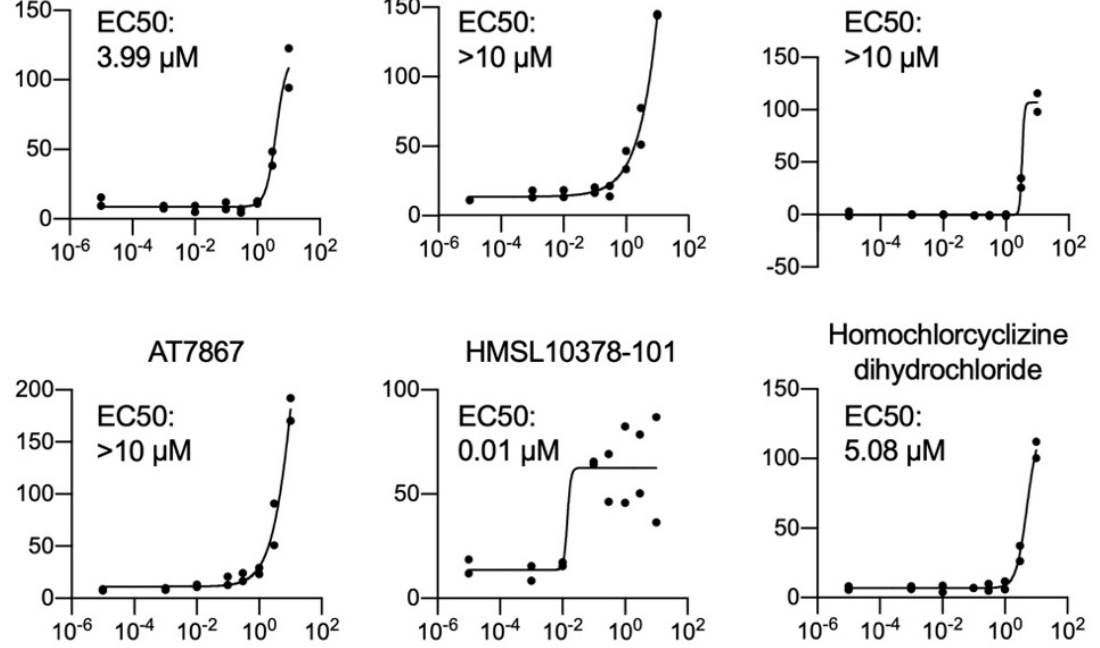

LY2608204

EC50:
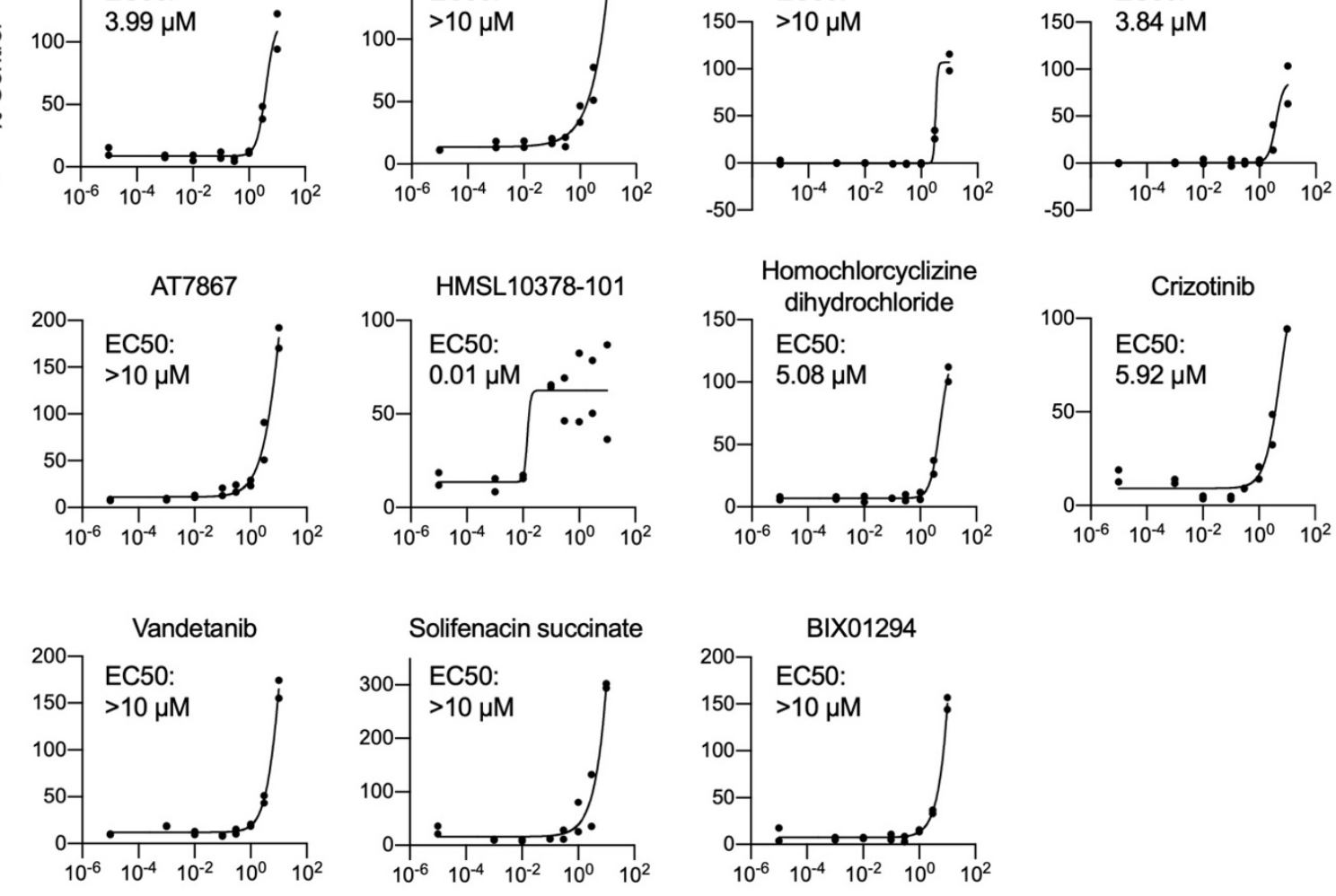

Concentration $(\mu \mathrm{M})$

Figure 1 - figure supplement 2. Nineteen compounds were identified to induce lipid accumulation in HSCs.

HSCs from human donor 2 were treated with compounds at indicated concentrations. Each dot represents one well ( $\mathrm{n}=2$ technical replicates per concentration). Curves were generated by fitting the data into a sigmoidal equation. For digitoxigenin and digoxin, the highest two or three concentrations respectively were not used for fitting the curves due to the toxicity at these concentrations. If a compound was tested more than one time, only one representative curve is shown here, while the average score from all curves was used for ranking the compounds. Results from each individual experiment and data from all replicates are in Supplementary Table 6. 

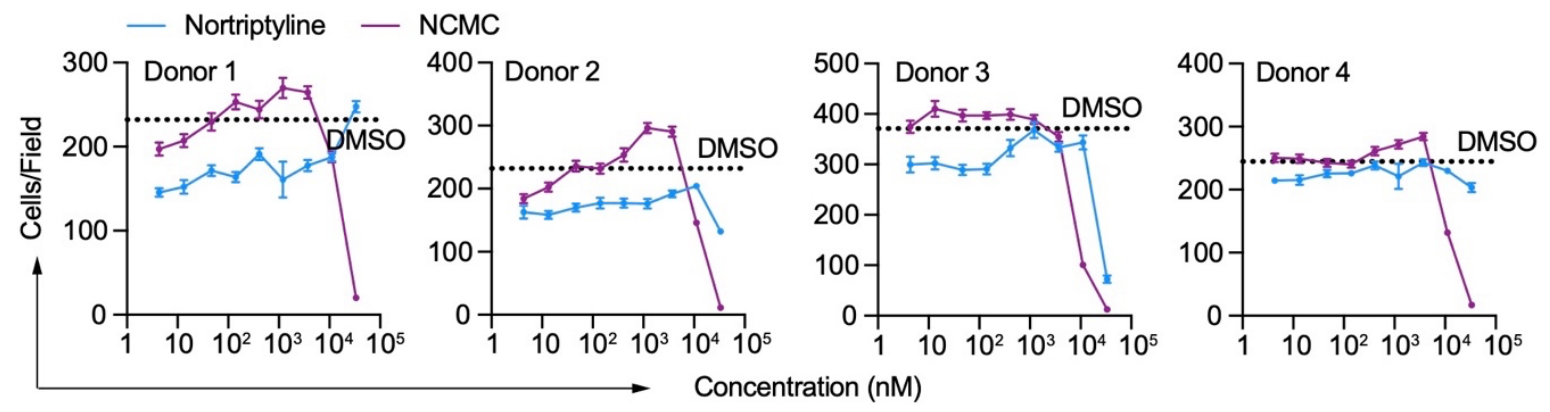

Figure 2 - figure supplement 1. Effect of NCMC on cell number

Effects of NCMC (purple) and nortriptyline (blue) on cell numbers in HSCs isolated from four different human donors at 48 hours. Dotted line represents the mean number of cells per microscopic field in DMSO control wells. Error bars represent mean \pm SEM $(n=12)$. One experiment was performed in each of the four donors. 
Human Donor 1
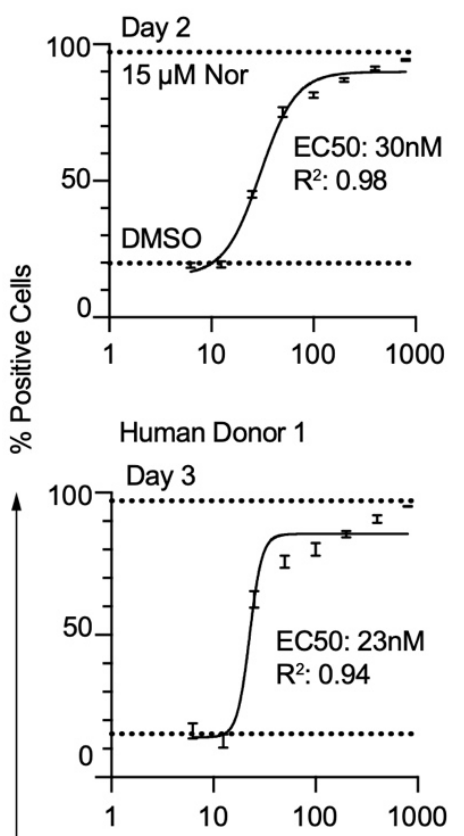

Human Donor 3

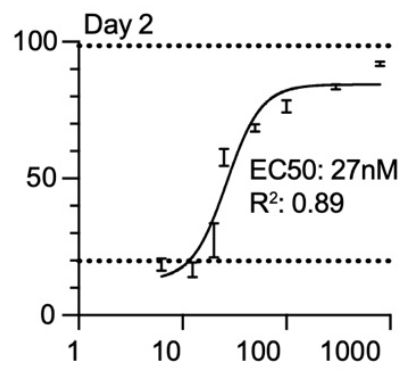

Human Donor 3

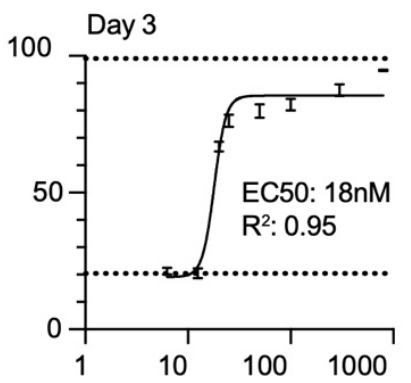

Mouse HSCs

100

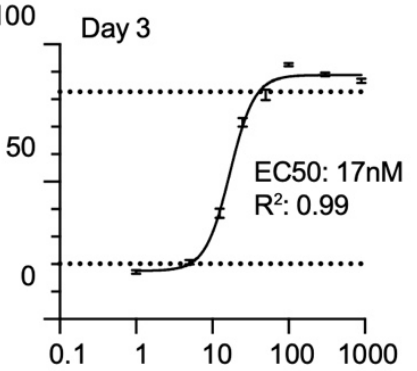

Mouse HSCs

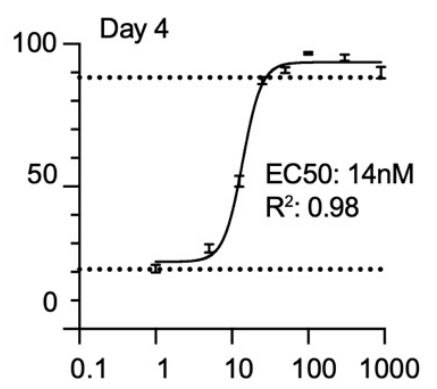

Nanchangmycin concentration (nM)

Figure 2 - figure supplement 2. Dose response curves of NCMC treatment at different time points in HSCs.

HSCs isolated from human donor 1 (left two panels), human donor 3 (middle two panels) and mouse (right two panels) were used. Dotted line represents the mean percentage of Bodipypositive cells in DMSO negative control wells (lower) or nortriptyline positive control wells (upper). Error bars represent mean $\pm \operatorname{SEM}(n=6)$. EC50 and $R^{2}$ are shown. 

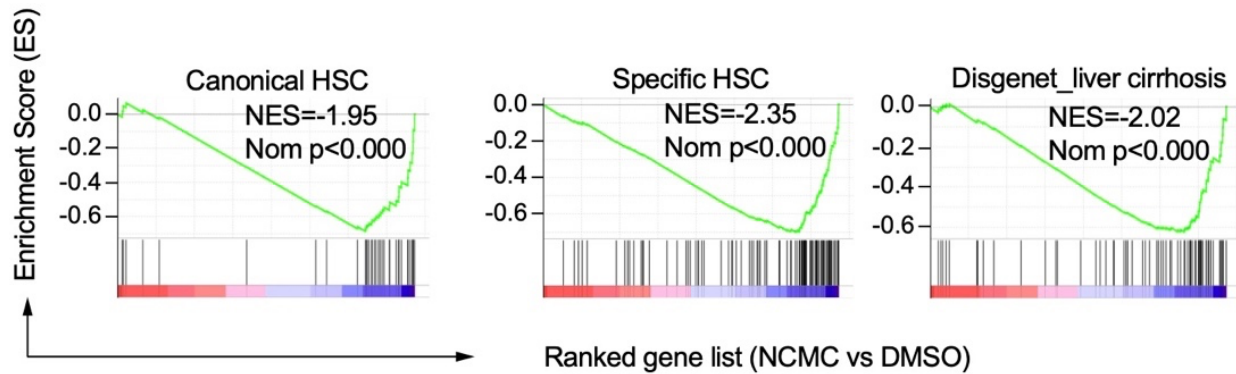

Ranked gene list (NCMC vs DMSO)

1250 Figure 3 - figure supplement 1. Gene set enrichment analysis results of HSC (Zhang et al., 2016) and cirrhosis (Piñero et al., 2020) gene signatures NES refers to Normalized Enrichment Score. Nom P refers to Nominal P value. Vertical black lines refer to the gene hits in the indicated signatures. Red color indicates positively correlated, and blue color indicates negatively correlated. 

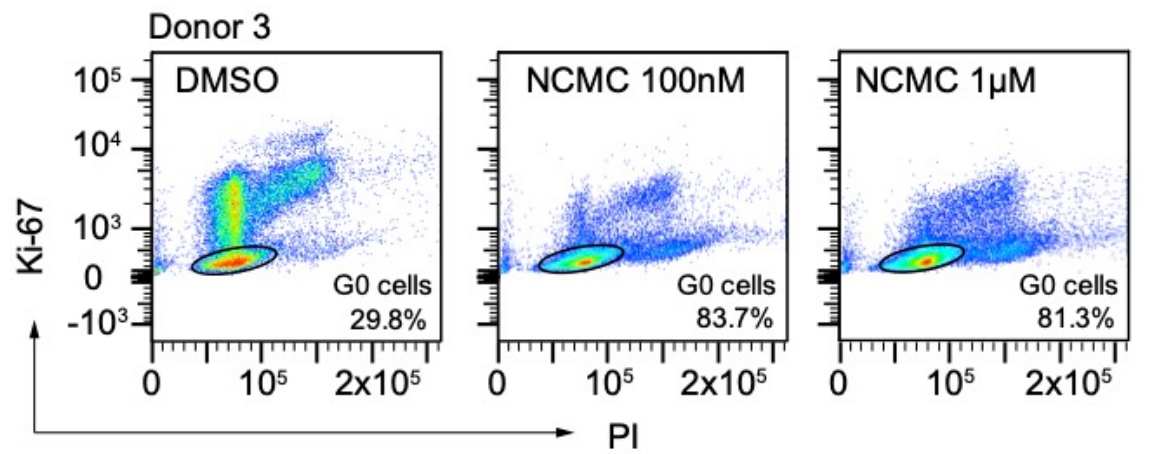

Figure 4 - figure supplement 1. Flow cytometry analysis of Ki-67 and PI stained HSCs HSCs from human donor 3 were treated with DMSO or nanchangmycin (NCMC) for 24 hours prior to flow cytometry analysis with Ki-67 and PI. Plots are representative of two independent experiments. 
A

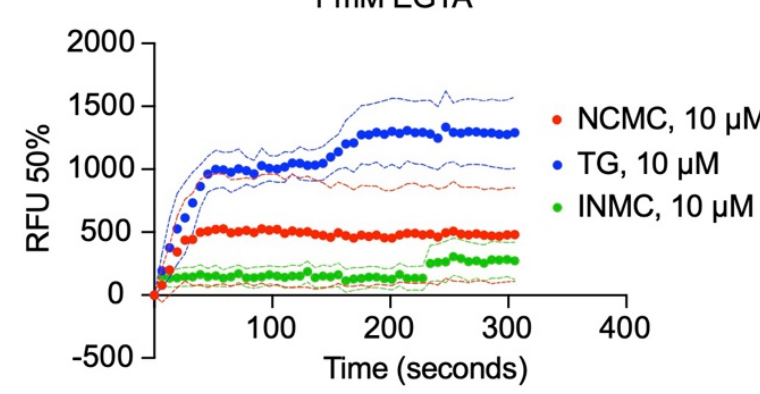

B

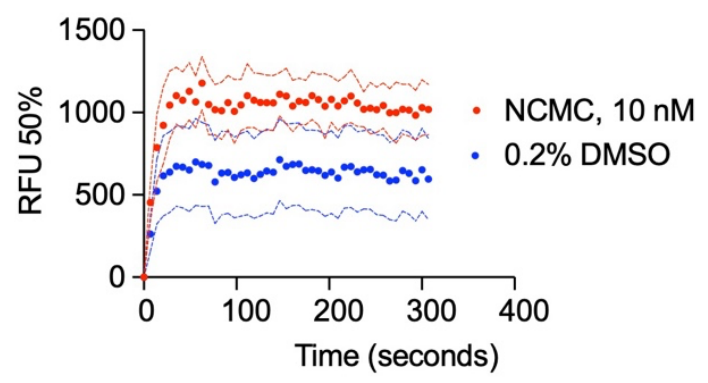

Figure 4 - figure supplement 2. Measurement of cytosolic calcium level using fluo-4

A. HSCs from donor 3 were pre-loaded with fluo-4 NW and fluorescent intensity was read immediately after adding compounds in the presence of $1 \mathrm{mM}$ EGTA (NCMC: nanchangmycin (red), TG: thapsigargin (blue), INMC: ionomycin (green)). The plot demonstrates results from three independent experiments. Solid dots represent mean, and dotted lines represent SEM $(n=3)$. RFU: Relative fluorescence unit. B. HSCs from donor 3 were pre-loaded with fluo-4 NW and fluorescent intensity was read immediately after adding $0.2 \%$ DMSO or $10 \mathrm{nM} \mathrm{NCMC}$ dissolved in DMSO (without EGTA). The plot demonstrates results from three independent experiments. Solid dots represent mean, and dotted lines represent SEM ( $n=8$ for DMSO, $n=4$ for NCMC). DMSO at $0.2 \%$ was selected as a negative control, as this was the highest concentration of DMSO to which HSCs were exposed, as a solvent. NCMC, TG and INMC were all dissolved in DMSO. 


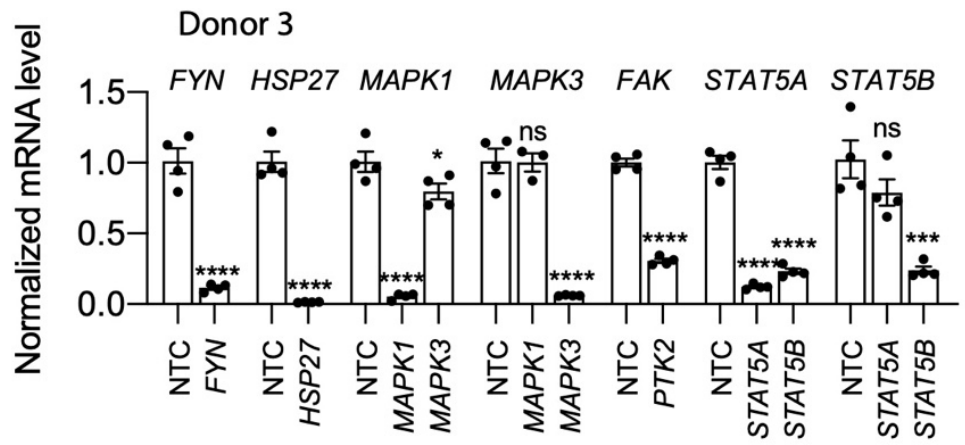

Donor 4

Figure 5 - figure supplement 1 . Knockdown efficiency of each siRNA pool in HSCs from human donor \#3 (top) and donor \#4 (bottom) compared to a non-targeting siRNA control (NTC)

Error bars represent mean \pm SEM $(n=4)$. One experiment was performed for each donor shown. ns indicates not significant $(p>0.05)$, ${ }^{*}$ indicates $p<0.05$, ${ }^{* *}$ indicates $p<0.01$, ${ }^{* * *}$ indicates $p<0.001$, and ${ }^{* * * *}$ indicates $p<0.0001$ (one-way ANOVA test). 


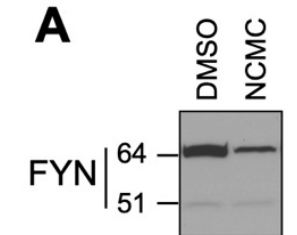

FYN (longer

$\beta$-actin
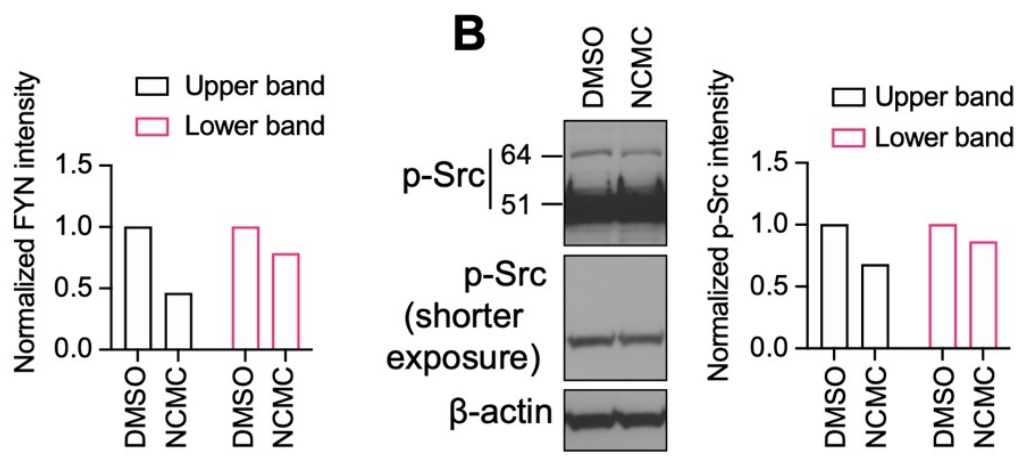

Figure 6 - figure supplement 1. Western blot of HSCs treated with $1 \mu \mathrm{M}$ NCMC for 18 hours

The same samples were loaded for $A$ and $B$ on the same gel. The membrane was cut and incubated with FYN and p-Src family antibodies separately. Bands were detected for FYN at 64 kilodaltons (kD) (Upper band) and $51 \mathrm{kD}$ (Lower band). Two different exposure times are shown for FYN in (A) and $p$-Src $(B)$ to evaluate both bands. $\beta$-actin is used as a loading control. (Data are representative of three independent experiments). 


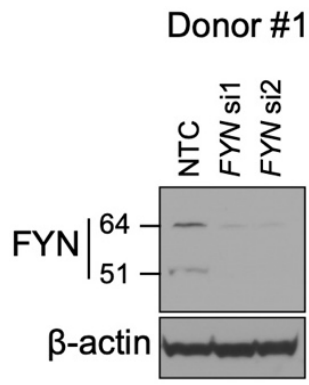

Donor \#3

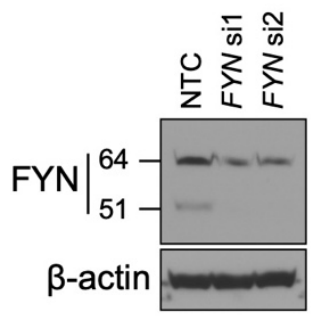

$\beta$-actin
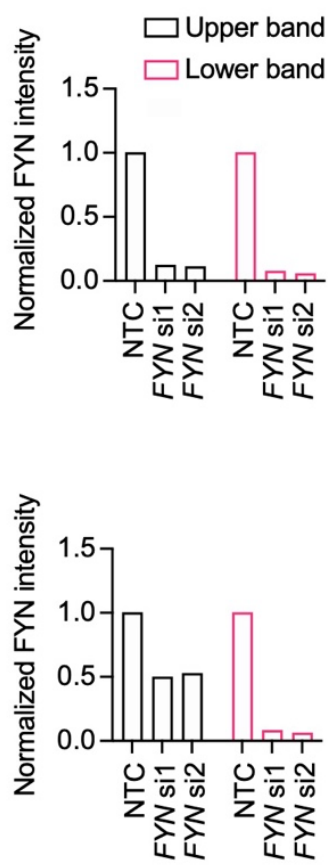

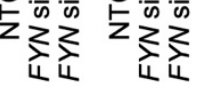

近这昰
1293

1294

1295

1296

1297

\section{Figure 6 - figure supplement 2. Western blot for FYN}

HSCs were transfected with siRNAs targeting FYN (si1 and si2) and a non-targeting siRNA control (NTC). Western blot was performed on day 3 (representative of two independent experiments). 


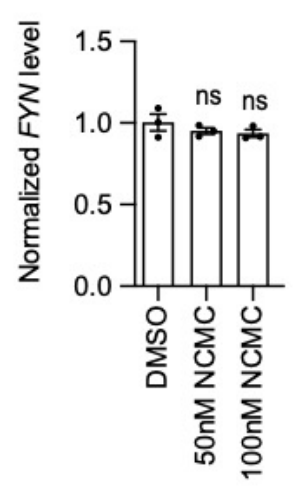

Donor 3, 2D

Figure 6 - figure supplement 3. FYN mRNA level was not affected by NCMC treatment HSCs were treated with NCMC for indicated number of days (D). FYN level was analyzed by qPCR. Error bars represent mean $\pm \operatorname{SEM}(n=3)$. Data are representative of two independent experiments. ns indicates not significant $(p>0.05)$ by one-way ANOVA for donor 1 and Student's t-test for donor 3. 
bioRxiv preprint doi: https://doi.org/10.1101/2021.10.08.463221; this version posted October 9,2021 . The copyright holder for this preprint (which was not certified by peer review) is the author/funder, who has granted bioRxiv a license to display the preprint in perpetuity. It is made available under aCC-BY-NC-ND 4.0 International license.

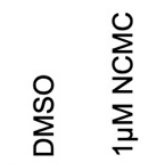

p-FAK

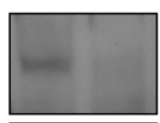

$\beta$-actin

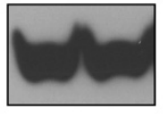

Figure 6 - figure supplement 4. NCMC decreases FAK phosphorylation Western blot of HSCs treated with DMSO control or $1 \mu \mathrm{M} \mathrm{NCMC} \mathrm{for} 18$ hours. $\beta$-actin is used as a loading control (representative of three experiments). 


\section{List of supplementary tables}

1311 Supplementary Table 1_Small-molecule Compound Libraries

1312 Supplementary Table 2_Primary Screen Results

1313 Supplementary Table 3_711 Hits from Primary Screen

1314 Supplementary Table 4_Clustering of Primary Screen Candidates

1315 Supplementary Table 5_Secondary Screen Results

1316 Supplementary Table 6_DRC Screen Results

1317 Supplementary Table 7_GSEA of Ontology Gene Sets

1318 Supplementary Table 8_HSC and Liver Cirrhosis Gene Signatures 